\title{
Sciences sociales et histoire
}

\section{Giovanni Busino}

\section{OpenEdition \\ Journals}

Édition électronique

URL : http://journals.openedition.org/ress/515

DOI : $10.4000 /$ ress. 515

ISSN : 1663-4446

\section{Éditeur}

Librairie Droz

\section{Édition imprimée}

Date de publication : 1 décembre 2003

Pagination : 119-171

ISBN : 2-600-00912-4

ISSN : 0048-8046

Référence électronique

Giovanni Busino, "Sciences sociales et histoire », Revue européenne des sciences sociales [En ligne], XLI-127 | 2003, mis en ligne le 30 novembre 2009, consulté le 19 avril 2019. URL : http:// journals.openedition.org/ress/515; DOI : 10.4000/ress.515 


\section{Giovanni BUSINO}

\section{SCIENCES SOCIALES ET HISTOIRE*}

SOMMAIRE: 1. Une collaboration difficile. 2. Un dialogue inextricable. 3. Les débats et les controverses contemporains. 4. Des problématiques communes. 5. Les apports de l'histoire. 6. Les apports des sciences sociales. 7. En guise de conclusion interlocutoire.

\section{UNE COLLABORATION DIFFICILE}

L'histoire, la sociologie et les autres sciences sociales ont pour référent et objets communs le «cours historique du monde», mais les analysent-elles de la même manière? Le cas échéant, peut-on identifier les spécificités des différentes approches utilisées?

Les réponses à ces questions renvoient souvent aux différences entre les disciplines produites par la division du travail académique, aux constats que les concepts utilisés, les perspectives adoptées, les modalités descriptives ou explicatives, les diversités des traditions intellectuelles rendent difficiles les transferts des acquis entre les sciences humaines, ils compliquent les emprunts et les échanges, entravent la coopération et révèlent pourquoi l'interdisciplinarité reste un horizon inaccessible.

$\mathrm{Au}$ lieu de broder ces arguments théoriques, examinons la problématique en partant des recherches consacrées à la «transition» et des approches adoptées pour l'étudier par les différentes disciplines de l'homme et de la société.

Il est notoire que les premières études sur la «transition ${ }^{1}$, c'est-à-dire sur le passage et les transformations d'un type de société à un autre, remontent à l'époque d'Adam Smith, de David Ricardo et des philosophes du XVIII' siècle. Ils ont été les premiers à décrire les effets et à expliquer les causes des bouleversements qui ont détruit les sociétés d'Ancien Régime et conduit à l'instauration des sociétés modernes. Karl Marx a repris, entre 1846 et 1876 , ces études et les a élaborées en un schéma explicatif du fonctionnement et de l'évolution des différentes formations économico-sociales s'étant succédées dans le cours de l'histoire

* Cet article a bénéficié des commentaires et des remarques de Jacques Coenen-Huther, JeanClaude Passeron et Philippe Steiner, auxquels vont ma gratitude et mes remerciements.

1 Lire R. Romano, Le problème de la transition du féodalisme «at present» dans l' cuvre d'Adam Smith, «Revue européenne des sciences sociales », XXXIV, 1996, n. 106, pp. 17-24 et G. Busino, La permanence du passé. Questions d' histoire de la sociologie et d'épistémologie sociologique, Genève, Droz, 1986, pp. 49-65. 
de même que de la correspondance entre les modes de production et les formes des rapports sociaux. Il a cru dévoiler ainsi la loi générale qui gouverne la naissance, le développement, la transformation, le déclin, la mort des sociétés.

Depuis, les travaux des historiens, des économistes, des politologues et des sociologues sur la «transition» des sociétés traditionnelles aux sociétés industrielles, sur le passage d'une forme sociale à une autre, ont proliféré sans pour autant que les connaissances se soient accrues. Les travaux d'une historiographie à l'instrumentation statistico-mathématique sophistiquée, les études sociologiques et politologiques sur la modernisation, n’ont pas révélé les «logiques», les «causes », les « raisons » qui permettent la reproduction d'un système économicosocial ni même les mécanismes qui favorisent la naissance d'une nouvelle organisation des rapports sociaux et l'aménagement de ceux-ci en une forme générale de société nouvelle.

Les explications de Marx sur le passage de la société féodale à la société industrielle capitaliste et ses lois régissant le mouvement économique de la société demeurent toujours la référence fondamentale, bien qu'elles négligent les questions de la transformation des régimes politiques, les idéologies et les structures de parenté.

L'analyse des lois du mouvement économique n'étant réalisable que sur la longue durée, Marx doit classer les matériaux historiques dans un ordre chronologique qui va de la Révolution anglaise de 1640 à la Révolution française de 1789. Une attention particulière est réservée à l'Angleterre où la vie matérielle a été façonnée par les rapports capitalistes de production, notamment dans les domaines industriels et agricoles. Ensuite, la distinction entre la genèse des rapports capitalistes de production et le développement de ces mêmes rapports lui sert à élaborer une périodisation en trois époques.

La première se situe entre le $\mathrm{XIV}^{\mathrm{e}}$ et le $\mathrm{XV}^{\mathrm{e}}$ siècle et se caractérise par la crise de la production féodale et l'abolition du servage. A la fin de cette période il y a une augmentation du nombre des paysans libres, la prospérité des villes et des petits propriétaires terriens. Des changements radicaux commencent à affecter l'agriculture: de nouvelles formes de propriété foncière apparaissent ainsi que de nouvelles méthodes de production et la naissance des manufactures pour l'exportation. L'augmentation du nombre des travailleurs libres active le déclin et puis la disparition du système féodal.

La deuxième va du XVIe à la première moitié du XVIII siècle. La révolution agricole se poursuit, la dépréciation des métaux précieux fait chuter le taux des salaires et le niveau de la rente foncière. L'augmentation du prix des marchandises enrichit les fermiers. Dans la même époque on observe le développement du régime colonial, du crédit, de la finance et l'avènement des premiers systèmes protectionnistes. Tous ces facteurs, agencés en diverses combinaisons, on les observe surtout en Angleterre, dès les années 1660, début de la révolution agricole. Les progrès de la révolution industrielle et du développement du machinisme sont si rapides et puissants, dès 1750 , que les petits propriétaires terriens en sont les victimes. C'est ainsi qu'à partir de la fin du XVIIe siècle, l'Angleterre supplante les Pays-Bas et devient le premier pays industriel du monde.

Pendant la troisième époque, de la fin du XVIII ${ }^{\mathrm{e}}$ au début du $\mathrm{XIX}^{\mathrm{e}}$ siècle, advient la structuration du mode de production capitaliste et la consolidation de sa base. Le machinisme et la grande industrie prospèrent; des formes spécifiques 
d'exploitation de la nature et d'accès aux ressources sont développées; des nouveaux modes d'organisation des processus de travail sont mis en place; la production et la redistribution des fruits du travail social. C'est précisément en cette période que les rapports de production deviennent aussi des rapports sociaux, qu'au mode de production vont correspondre des formes déterminées de parenté, de gouvernement, de pensée et de représentation du monde. Les rapports entre la structure et la superstructure forment une totalité organique et la production matérielle, dans une société donnée va dépendre de l'articulation des formes sociales avec le mode de production économique.

La démarche régressive permet de remonter de la structure des rapports de production capitalistes aux conditions historiques de leur genèse, de construire des généalogies, de sélectionner, dans l'ensemble des pratiques sociales et des événements du passé, les facteurs déterminant la situation présente. Ce procédé régressif s'accompagne d'une approche développementale-constructiviste qui explicite la transition de la forme unique dominante vers les différentes formes de production du présent.

Cela ne constitue pas une théorie du mode de production féodal, ni même une bonne formulation des lois économiques de la transformation ou de la dissolution des différentes formes de propriété et de production féodales. Tout au plus Marx nous fournit quelques indications sur la rente foncière, sur les formes économiques rattachées aux rapports de propriété féodaux, sur la manière dont ces rapports commencent à se défaire dès que la rente en travail et en marchandise se transforme en rente monétaire. La formulation marxienne met également en évidence le phénomène de l'accès des paysans à la propriété des terres, les modes de production basés sur la petite propriété, la transformation des droits féodaux en des rapports capitalistes dans la nouvelle agriculture. Ainsi est amorcée l'analyse de l'accumulation primitive du capital dans le mode de production capitaliste. Certes, la production de marchandises, la propriété privée, l'utilisation de l'argent comme capital, le travail salarié ont existé auparavant, cependant ils ne se sont étroitement imbriqués que dans les sociétés capitalistes. Pour cette raison l'étude de leur genèse est moins intéressante que celle des conditions de leur généralisation actuelle. L'analyse de la décomposition d'un mode de production et d'une formation économico-sociale, de la dissolution des anciens rapports et de la constitution des nouveaux, vise à démontrer que les contradictions entre la structure et la superstructure engendrent une nouvelle base matérielle et de nouveaux rapports sociaux.

Pour Marx la transition se réduit aux processus de formation d'une nouvelle totalité organique, dont les mécanismes fondamentaux sont essentiellement les relations sociales des rapports de production agricole qui rendent possible l'accumulation primitive du capital.

La plupart des historiens considèrent que cette théorie du mode de production capitaliste est en contradiction avec la succession des formes de la rente du travail salarié, du commerce et du capital financier. Convaincu que la succession historique des faits n'explique rien, que la description d'un nouveau mode de production, des facteurs qui le déterminent, l'analyse de la nouvelle organisation sociale et de la succession de ses diverses composantes ne rendent pas compte du passage d'une société à une autre, Marx se propose d'élucider préalablement les raisons ou les logiques qui régissent la succession observée. Cependant dans cette approche 
il n'y a aucune place pour le rôle des systèmes de parenté, pour les transformations des structures familiales lors de la genèse et de la consolidation des modes de production. Les facteurs susceptibles de rendre compte de la transformation des superstructures, des rapports non économiques et, en particulier, des formes de pensée élaborées et développées dans le mouvement de la croissance du capitalisme sont tous négligés. Le rôle des systèmes d'idées et des mentalités dans la formation et dans la transformation des rapports sociaux y sont insignifiants tandis que les rapports entre le centre et la périphérie à l'intérieur d'un mode de production donné y sont ignorés.

Marx n'a pas laissé une théorie bien formée de la transition d'une formation économico-sociale féodale à une formation économico-sociale bourgeoise. Il en est de même de celle de révolution industrielle qui reste inapte à rendre compte de la transition du féodalisme au capitalisme. Les conceptualisations élaborées jusqu'ici sur cette base demeurent toutes fragiles. En effet, elles doivent utiliser synonymiquement les concepts de croissance et de développement, de développement capitaliste et d'industrialisation, de processus d'industrialisation et de processus de modernisation, de modernisation et d'industrialisation, d'industrialisation et de capitalisme.

Certaines interprètes ont tenté de contourner ces difficultés. Les plus importantes de ces tentatives sont deux. La première est l'oeuvre d'historiens plus ou moins convaincus de la vanité, sinon de l'impossibilité d'élaborer une théorie réaliste de la transition; la seconde est l'oeuvre d'économistes, des sociologues et d'anthropologues persuadés de la nécessité d'une telle théorie.

Comme on le sait, les historiens ont étudié la nature du capitalisme dès le XIV ${ }^{\mathrm{e}}$ et jusqu'au XIX $\mathrm{X}^{\mathrm{e}}$ siècle, et ont analysé ses particularités, ses différences et ses analogies d'une époque à l'autre, d'une région à l'autre. Ils se sont gardés jusqu'ici de tirer de tous ces travaux une vision synthétique, voire une théorie générale. Les exemples les plus remarquables se trouvent dans les travaux de F. Braudel ${ }^{2}$ et de P. Bairoch ${ }^{3}$.

Le premier a brossé un tableau du capitalisme européen caractérisé par sa créativité excentrique, par son pouvoir de se soustraire aux conditionnements et aux uniformités, par une énergie quasi naturelle. Le second, au contraire, s'est efforcé de repérer et de reconstruire les facteurs à l'origine du développement (le progrès technique, les facteurs démographiques, la montée des prix, l'accumulation du capital) et en conclut que ce dernier a été engendré par les progrès de l'agriculture antérieurs à ceux du secteur industriel: «[...] il est [...] possible d'affirmer que non seulement l'accroissement de la productivité agricole a été le facteur déterminant de l'amorce de l'industrialisation, mais qu'un accroissement sensible, tant en ampleur qu'en durée, de la productivité agricole a dû, dans la plupart des cas, provoquer une amorce du processus d'industrialisation, et ceci aussi longtemps que les progrès de la médecine n'ont pas permis à la poussée démographique d'absorber la totalité du bénéfice de la variation de la production agricole résultant de ce progrès ».

F. Braudel, Les ambitions de l' histoire. Edition établie et présentée par R.de Ayala et P. Braudel. Préface de M. Aymard, Paris, de Fallois, 1997.

P. Bairoch, Victoires et déboires. Histoire économique et sociale du monde du XVIe siècle à nos jours, Paris, Gallimard, 1997, 3 vols. 
L'agriculture a engendré ce phénomène en boule de neige grâce aux effets directs et indirects de l'accroissement de la demande, aux interactions dérivant de ces effets, directs et indirects, qui ont constitué les mécanismes de diffusion ou d'entraînement, les mécanismes fonctionnels grâce auxquels l'industrialisation a été réalisée.

Si chez F. Braudel et chez I. Wallerstein ${ }^{4}$ il y a une phénoménologie du capitalisme et de l'économie-monde conçus comme force indispensable au mouvement sociétal, P. Bairoch élabore un modèle des principaux mécanismes économiques mis en place durant de la révolution industrielle, des rapports qui les unissent, des effets induits par leurs interactions. De ce modèle, par déduction logique, est dérivée la transformation de l'économie et de la société. Quant aux «social scientists », ils ont tantôt dénoncé les lacunes et les négligences du modèle marxiste et tantôt essayé de le compléter ou de lui opposer d'autres modèles, tel celui de W. W. Rostow ${ }^{5}$. Cependant, ils sont tous partisans d'une explication globale et unitaire, bien que la nature de leurs explications varie passablement. Certains s'attachent à mettre en évidence les forces endogènes à l'origine de la transition, comme par exemple John Hicks ${ }^{6}$ qui l'identifie au marché. D'autres se tournent vers les forces exogènes: A. O. Hirschmann ${ }^{7}$ considère que c'est la mutation du système des valeurs éthiques et politiques qui a modifié le regard sur les phénomènes économiques et les activités de commerce et qui a fait de la poursuite de l'intérêt une règle de conduite mobilisatrice des passions. En effet, le capitalisme serait parvenu à maîtriser les passions destructrices en les canalisant dans la cupidité et l'appât du gain, donc en tuant les passions, en détruisant le mystère et la magie, en supprimant l'indifférence. Et c'est cette mutation qui serait à l'origine du processus de transition de l'économie féodale à l'économie capitaliste.

Le moment le plus crucial de ce conflit entre historiens, économistes et sociologues se situe au lendemain de la publication des études de Maurice Dobb ${ }^{8}$ sur le développement du capitalisme, avec lesquelles ce chercheur se propose de démontrer que la thèse de Marx est vérifiée par les acquis de la recherche historique moderne.

Dans ses travaux Dobb passe en revue, à la lumière d'un important matériau historique, le concept de féodalité, les origines de la bourgeoisie, l'émergence du capital industriel, l'accumulation du capital, le mercantilisme, la croissance industrielle, la révolution industrielle et la période subséquente. Les conclusions

I. Wallerstein, The Capitalist World-Economy, Paris, MSH, 1979; The Politic of the WorldEconomy, Paris, MSH, 1984; Le capitalisme historique, Paris, La Découverte, 1985.

W. W. Rostow, The Stages of Economic Growth, Cambridge, Cambridge University Press, 1960.

6 J. Hicks, A Theory of Economic History, Oxford, Clarendon Press, 1961, et Causality in Economics, London, Blackwell, 1979.

A. O. Hirschman, Essays in Trespassing Economics to Politics and Beyond, Cambridge, Cambridge University Press, 1981, et Come complicare l'economia, Bologna, Il Mulino, 1988, mais surtout The Passions and the Interests. Political Arguments for Capitalism before its Triumph, Princeton, N.J., Princeton University Press, 1977.

s M. Dobb, The Transition from Feudalism to Capitalism, London, New Left Books, 1976 et Prelude to the Industrial Revolution, «Science and Society», Vol. XXVIII, n. 1, Winter 1964, pp. 31-47. Voir aussi le recueil: M. Dobb, R. Hilton, E. Hobsbawm, A. Maczak, F. Mazzei, J. Merrington, A. Soboul, I. Wallerstein, Dal feudalismo al Capitalismo, Napoli, Liguori, 1986. 
auxquelles ce chercheur anglais semble parvenir peuvent être résumées ainsi: il s'est produit une concentration de la propriété foncière entre les mains des seigneurs et des paysans riches aux dépens de la petite paysannerie. Ce processus de concentration d'une part et d'expropriation de l'autre a pour conséquences: (a) l'accumulation de capitaux dans les mains de la paysannerie, dont une part sera investie dans l'industrie; (b) la formation d'un marché intérieur: les paysans expropriés doivent acheter ce qu'auparavant ils produisaient eux-mêmes; de même, les paysans capitalistes doivent acheter les biens de consommation et les moyens de production; (c) les paysans expropriés constituent une main d'œuvre à la fois agricole et industrielle. La naissance de la société capitaliste est donc favorisée par la constitution du marché, par la formation d'une main d'œuvre abondante et par la disponibilité de capitaux. Ces éléments entraînent la différenciation entre la classe des propriétaires et celles des travailleurs, leur antagonisme, et par là la lutte des classes. Le mode de production capitaliste est ainsi constitué.

Ces thèses ont suscité des controverses aussi passionnées qu'interminables. Alors que les travaux de Hicks et de Hirschman ont été jugés des exercices théoriques sans rapport avec l'histoire, les historiens se sont montrés fort prudents à l'égard des ceux de Dobb. Tout en reconnaissant que celui-ci maîtrise les résultats des enquêtes historiques, les résultats auxquels il arrive ont été jugés prématurés. Ils généralisent à l'excès des données locales et spécifiques, des particularités et des individualités sectorielles.

La problématique la plus controversée concerne l'Italie du $\mathrm{XV}^{\mathrm{e}}$ et du XVI ${ }^{\mathrm{e}}$ siècles, qui n'a aucune place dans le modèle de Dobb, entièrement construit sur la comparaison des développements anglais et français. Dans un modèle à prétention globalisante trouvent une systématisation exclusivement les «réussites» et les «gagnants». Ceux qui n'ont pas « réussi », qui ont «pris du retard», qui n'ont pas voulu ou pas pu «réussir» sont absents. Le modèle ignore également ceux qui avaient au départ une position favorable mais ont été dans l'impossibilité d'en profiter (c'est le cas de l'Italie du XVIe siècle) et ceux qui se sont révélés incapables de réaliser la transition (les Pays-Bas du XVII ${ }^{\mathrm{e}}$ siècle).

Ce débat a été décevant car il a été cantonné à l'analyse du servage et de l'organisation de la production de la propriété seigneuriale, ou à l'étude de l'articulation originaire entre producteurs directs et propriétaires terriens, de l'étude du développement du commerce et de la production pour le marché. N'arrivant pas à sortir de la perspective exclusivement économique, la prise en compte des «extravagances » de l'Italie de la Renaissance et des Pays-Bas du XVII ${ }^{\mathrm{e}}$ siècle était impossible. La vision économique du passage de la société traditionnelle à la société moderne, la recherche d'une explication généralisante basée sur les seuls facteurs économiques, le cas anglais considéré paradigmatique, tous les autres étant non pertinents, ont conditionné les débats. La transition en tant que phénomène essentiellement économique s'étant manifesté en sa plénitude surtout en Angleterre, sur la politique commerciale et coloniale, sur les élites attirées par le gain, sur une culture légitimant la réalisation de l'intérêt et sur des développements parallèles (et jamais subordonnés) de l'économie rurale et agricole et d'une économie non agricole, oblige à faire des analyses en termes de séries d'équilibres socio-économiques mouvants, donc des processus continus, linéaires. L'économie est le moteur de la transition et cette dernière est l'indicateur premier de la transformation générale de la société. Ceci explique pourquoi on n'a pas pu parler 
d'évolution mais seulement de révolution. Ceci explique également l'importance primordiale qu'ont dans ce modèle les tensions, les conflits, les contradictions et les rapports d'antagonisme, tout comme la nécessité d'identifier l'agent historique du changement, d'ignorer les résistances, les obstacles et les refus que ce même sujet de l'action historique oppose aux innovations et à la modernisation. C'est pourquoi, enfin, il a fallu gommer les discontinuités et ne mettre en évidence que les continuités, réduire les spécificités de chaque société, leurs formes particulières à des caractéristiques secondaires ou non pertinentes. La transition en tant que processus économique implique l'autonomie de l'économique et ses déterminismes. Or ni les forces du marché, ni l'offre de main-d'œuvre, ni l'adoption de nouvelles techniques, agricoles ou autres, ni les données monétaires ne constituent des conditions nécessaires et suffisantes pour assurer le passage d'une forme de société à une autre. Le réductionnisme économique dissimule le fait que l'économie est toujours subordonnée aux facteurs socioculturels et politiques, ainsi que les travaux de W. Kula l'ont prouvée.

Les résultats obtenus. Ils ne fournissent pas d'éclaircissements sur les problèmes du présent, sur les différentes évolutions historiques de sociétés pourtant comparables entre elles. Une théorie de la transition bien formée devrait pouvoir élucider une série de questions telles, par exemple, que celles-ci: (a) pour postuler l'antériorité de la dimension économique dans le processus de transition, il faudrait au préalable savoir si le commerce et la finance sont bien à l'origine de l'accumulation primitive du capital, ou bien si c'est le progrès agricole et la croissance démographique qui donnent l'impulsion au développement de la protohistoire; (b) pourquoi tous les grands centres urbains de l'époque pré-industrielle perdent leurs rôles et leurs fonctions au fur et à mesure de l'industrialisation? Estce dû à un système économique et social qui, au cours des phases de transition, se situe entre le système urbain du commerce et de l'artisanat et celui du capitalisme industriel naissant?; (c) les économistes affirment qu'il existe des facteurs objectifs expliquant les retards persistants et les rattrapages. Les groupes humains mettent en œuvre face à des circonstances identiques des solutions différentes. S'il est possible faire un choix entre différents facteurs, le système n'est ni mécanique ni nécessaire, il est une fonction de rapport sociaux à identifier; $(d)$ si l'industrialisation s'identifie avec le capitalisme, est-ce que celui-ci est un système d'échanges basé sur le marché et la monnaie, ou bien est-il un système d'accumulation du capital fixe au détriment du capital circulant?; (e) pourquoi postuler que la maximisation est inhérente aux seuls comportements économiques capitalistes, alors qu'il a été possible de maximiser n'importe quel type de comportement dans n'importe quel système économique à partir des seules valeurs ou raisons signifiantes? Ce qui change dans l'économie capitaliste, ce sont les conditions et les contraintes, et donc les décisions, mais pas nécessairement les principes. Par exemple, l'accumulation et l'investissement peuvent se retrouver dans n'importe quel type de société. Ces deux éléments deviennent des fonctions déclenchantes si les milieux technologiques et socio-économiques se prêtent à un mode de produc-

Sur l'œuvre de l'historien polonais voir les travaux de M. Herling Bianco, Braudel e Kula. Un «incontro» fra due tradizioni storiografiche ed intellettuali, in: B. Angelo et G. Muto, Fernand Braudel : il mestiere di uno storico, Napoli, ESI, pp. 45-54, et La transizione dal feudalesimo al capitalismo nell' opera di Witold Kula, in «Società e Storia », n. 42, 1988, pp. 979-993. 
tion plus intense, qui valorise le profit monétaire. Il a fallu franchir un seuil décisif, celui de la transformation du travail humain en marchandise abstraite et de cette marchandise en objet d'échanges, pour que l'échange prenne la forme qu'on lui connaît dans les sociétés capitalistes; $(f)$ le passage d'un mode de production à un autre est un phénomène unique et irréversible. Les transformations qu'une société subit s'opèrent toujours à travers des équilibres précaires, multiples, variés et souvent aussi régressifs. Comment les identifier, les décrire et surtout les évaluer?

Cette problématique nous rappelle que: (A) Si le mode de production s'explique par rapport aux processus d'accumulation primitive alors il faut décrire et préciser en priorité les mécanismes de l'accumulation et ses orientations, quels sont les mécanismes ayant conféré au capital une mobilité extrême et si ces mécanismes proviennent des institutions financières. $(B)$ Pour importante qu'elle soit, l'analyse des forces productives est reliée aux rapports que les groupes sociaux établissent entre eux en fonction, notamment, de la propriété des moyens de production, de la répartition des ressources et des pratiques sociales. Comment étudier ces rapports dans les sociétés de l'époque préindustrielle? $(C)$ La participation à la détermination des pratiques sociales pose le problème de l'hégémonie, du pouvoir et de l'Etat. L'Etat serait né de la désintégration de la société féodale, entre 1280 et 1360, produit des guerres, de l'impôt et de la laïcité. L'Etat aurait créé un marché unifié, mais aurait aussi aboli le phénomène de la désertion d'un territoire par les populations, phénomène qui dans les sociétés sans Etat (comme, par exemple, chez les Nambikwara ou les Nuers) remplit la double fonction de désamorcer les conflits et de perpétuer un processus de migration et de transplantation continues ${ }^{10}$.

Dans les faits, les Etats des XVII' et XVIII ${ }^{\mathrm{e}}$ siècles empêchèrent la désertion ainsi que la conversion au protestantisme des individus et des groupes, mais jamais l'exode des capitaux, dont la mobilité était considérée, même par Montesquieu et par A. Smith, comme étant de nature à prévenir les «grands coups d'autorité » des souverains. Dès lors, ce que fut la véritable fonction de l'Etat dans le processus de transition reste à déterminer.

Les «nouveaux économistes» défendent un paradigme où l'explication économique est purifiée de l'antagonisme capital / travail et de la lutte de classe appliquée aux institutions. Inspiré par les «concepts économiques classiques», l'appareil conceptuel de ce paradigme est celui de la rationalité des choix humains. Douglass C. North ${ }^{11}$ est le plus célèbre théoricien de cette mouvance. Il attribue à chaque élément une autonomie relative dans le cadre d'un système d'interrelations, de sorte qu'il peut ramener toutes les explications sectorielles à une

o Voir à ce propos le recueil d'études L'esprit des lois sauvages, Paris, Seuil, 1988 ainsi que le livre d'A. Testart, Les chasseurs-cueilleurs, ou l'origine de l'inégalité, Paris, Société d'Ethnographie, 1982.

11 D. C. North, The Rise of the Western World, Cambridge, Cambridge University Press, 1973; Structure and Change in Economic History, New York, Norton, 1982; Institutions, Institutional Change and Economic Performance, Cambridge, Cambridge University Press, 1990; Transaction Costs, Institutions and Economic Performance, San Francisco, CA, ICS, 1992. Lire sur l'euvre de ce prix Nobel: R. Giannetti et A. Baccini, Un Nobel a sorpresa, «Storia del pensiero economico», n. 26, n.s., 1993, pp. 3-22. 
seule cause, la cause des causes, et c'est l'explication ultime. La thèse principale est formulée ainsi: «La Révolution industrielle n'est pas la cause de la croissance, elle n'est qu'une des manifestations, l'un des révélateurs d'un phénomène nouveau, la croissance économique, dont les origines remontent à des temps anciens et au cours desquels s'est lentement construite la structure des droits de propriété. C'est cette structure qui a créé les conditions d'un fonctionnement social favorable à un meilleur développement des ressources à la société». Ce type de phénomène serait intervenu tout d'abord aux Pays-Bas, «la première nation européenne à se doter d'un système d'institutions et de droits de propriété permettant d'exploiter de façon efficace les motivations individuelles pour assurer l'orientation des capitaux et des énergies vers des activités socialement les plus utiles ». Cela a été possible grâce à la mise en place d'une technologie performante d'organisation des rapports humains, économiques et sociaux. Cette technologie dérive de l'octroi des droits de propriété, droits consécutifs à la fin du pacte féodal. Lorsque la noblesse n'est plus parvenue à assurer la sécurité et la justice, le système de servage qui conférait le droit à l'usufruit a perdu sa raison d'être. L'équilibre a été rompu par la croissance démographique. La fin de l'usufruit féodal et du servage va donner naissance, en trois étapes, aux formes modernes de la propriété foncière. $\mathrm{Du} \mathrm{XI}^{\mathrm{e}}$ au XIII ${ }^{\mathrm{e}}$ siècle, il y a modification de l'économie du système de servage, car la terre est rare et le travail abondant. Les paysans acquièrent la pleine propriété de leur force-travail, c'est-à-dire qu'ils sont libérés de leurs obligations corvéables contre le paiement d'une taxe annuelle. Dès ce moment, le paysan demande au seigneur la location du domaine qu'auparavant il cultivait en ne payant que l'usufruit des produits du domaine seigneurial. Le droit héréditaire à l'usufruit, représenté par le travail gratuit du serf, est remplacé par la rente, sorte d'obligation monétaire contractuelle enracinée dans un droit d'exploitation foncière. Il ne s'agit pas encore de la rémunération d'un droit de propriété, mais c'est déjà sa genèse. Avec le temps, lentement, les rapports féodaux de suzeraineté se relâchent et libèrent la propriété de la forcetravail par le truchement de la taxe; ils délivrent également des obligations personnelles moyennant le paiement d'une somme forfaitaire. L'individu acquiert ainsi le droit de propriété sur sa force de travail personnelle, tandis que le vassal se dégage de ses obligations en versant un «impôt » à son suzerain. C'est ainsi que se met peu à peu en place un véritable droit de propriété seigneurial.

Dès la seconde moitié du XIII siècle la raréfaction des terres et la hausse des prix agricoles accélèrent le mouvement. L'économie nouvelle démantèle l'organisation basée sur le servage et ruine le pacte féodal. Bien qu'elle soit décimée par les famines et les épidémies, la population croît plus rapidement que la production. Les termes de l'échange favorisent davantage les paysans que les titulaires des droits féodaux. La durée des baux s'allonge. La précarité de la location disparaît lorsque le seigneur reconnaît la transmissibilité du droit de location d'une génération à l'autre moyennant le paiement d'une solde à chaque succession. Lorsque s'accentue au XVI ${ }^{\mathrm{e}}$ siècle le cycle malthusien d'expansion et que l'inflation en souligne la force, les termes de l'échange favorisent la montée des loyers fonciers au profit de la noblesse. La baisse des salaires fait disparaître les derniers résidus du servage, désormais improductif, puisque la main-d'œuvre salariée est beaucoup moins chère que les serfs. C'est la fin des institutions féodales, la naissance de la propriété foncière et aussi de la propriété individuelle. 
Pour combattre les effets de la dépression malthusienne du XVII ${ }^{\mathrm{e}}$ siècle, il faut augmenter le rendement des terres. Ce qui aboutira à la révolution agricole, prélude à la révolution industrielle. Les innovations technologiques ont été réalisables grâce aux droits de propriété. La définition rigide de la structure juridique du système foncier, dont le mouvement des enclosures est l'aspect le plus significatif, stimule les intérêts individuels et collectifs et incite à des choix dont la rationalité économique se révélera plus performante, donc plus rentable.

Le modèle proposé par North est une version nouvelle du modèle classique de Malthus et de Ricardo. Les variations de la population, les rendements croissants de la population agricole, le mouvement des prix relatifs en constituent les pièces maîtresses. L'accroissement de la population, et donc des besoins alimentaires, oblige à mettre en culture de nouvelles terres et à augmenter le rendement de celles qui le sont déjà. Mais les salaires diminuent puisque la main d'œuvre est abondante. Les prix des produits agricoles, en revanche, augmentent. Ce seront les famines et les épidémies qui rétabliront l'équilibre en diminuant la population, et donc la demande en produits alimentaires. Les prix suivront le même mouvement descendant. La main d'œuvre étant devenue rare, les salaires augmentent, alors que les ventes et les profits diminuent. La nouvelle répartition du revenu favorise l'augmentation de la population, et le cycle peut recommencer. Selon North, la logique du cycle a été rompue par l'introduction d'institutions nouvelles permettant une organisation économique plus efficiente, c'est-à-dire une meilleure utilisation du mouvement des prix relatifs. Les possibilités de changement sont par conséquent liées au système des prix, indicateur des raretés relatives de nature physique et donc générateur de comportements de résistance anticyclique. L'état des prix permet donc d'apprécier les situations et d'élaborer des réponses toujours plus adéquates. Lorsque les institutions favorisent ce processus, la transition pourra s'opérer. En d'autres termes, le passage d'une société à l'autre est assuré par le mode d'organisation des rapports sociaux. Selon que les institutions réglant l'organisation de ces rapports favorisent les choix en maximisant l'intérêt individuel et collectif, les décisions ultimes seront plus performantes. Parmi toutes les technologies mises en œuvre au service de la «performance» des rapports humains et sociaux, ce sont les droits de propriété qui ont permis aux sociétés occidentales de gérer au mieux le passage des sociétés traditionnelles aux sociétés industrielles. Pour conclure sur ce point, citons Harold Demsetz pour lequel «les droits de propriété permettent aux individus de savoir a priori ce qu'ils peuvent raisonnablement espérer obtenir dans leurs rapports avec les autres membres de la communauté. Les anticipations se matérialisent par les lois, les coutumes et les mœurs d'une société. Détenir des droits, c'est avoir l'accord des autres membres de la communauté pour agir d'une certaine manière et attendre de la société qu'elle interdise à autrui d'interférer avec ses propres activités, à la condition qu'elles ne soient pas prohibées. Les droits de propriété permettent à leur détenteur de faire du bien ou du tort aux autres membres de la société, mais pas n'importe quel bien, pas n'importe quel tort $»^{12}$.

12 H. Demsetz, Towards a Theory of Propriety Rights, «American Economic Review», 57, May 1967 , pp. $347-359$. 
Les droits de propriété définissent la manière dont les individus peuvent soit tirer un profit de certaines activités soit être pénalisés à cause d'elles. Ces droits déterminent par conséquent qui doit payer pour modifier les actions d'autrui. La reconnaissance de ces droits conduit à établir une relation assez étroite entre les droits de propriété et le contexte extérieur. Dans ces conditions le rôle et la place des organisations et des institutions qui permettent la transition et déterminent la performance de l'économie sont essentiels. Cette théorie néglige toutefois le fait que toute transition est en dernière analyse la solution d'un conflit et que dans un conflit nul ne peut être gagnant sans laisser derrière lui un perdant. Le marché, mécanisme indispensable au maintien des droits de propriété, n'est pas neutre, il est plutôt le reflet, la caisse de résonance des valeurs collectives relativement à la supériorité des droits de propriété.

Les économistes ont développé les concepts d'utilité, de préférence et de demande révélée pour expliquer le fonctionnement d'un système économique indépendamment des sentiments de ses participants. Sur le marché, le bien-être de tous serait maximisé sans que personne n'ait à se préoccuper préalablement du bien-être de son voisin. Or, la «main invisible» d'Adam Smith n'est pas un don spontané de la nature, mais bien le produit d'un ensemble de choix collectifs en faveur du droit de propriété, assorti d'un certain nombre d'auto-restrictions qui en sont le corollaire. En effet, le droit de propriété exercé par un individu sur un bien en limite la jouissance par autrui. Les ressources étant limitées et les désirs individuels infinis, la rareté relève donc d'un phénomène social et non d'un fait de nature. Il en découle que l'interdépendance des individus persiste, même lorsque le système des droits de propriété ferait croire à la liberté individuelle. Nous sommes privés, en quelque sorte, de ce que possèdent les autres, et réciproquement. Dans le même ordre d'idées, l'ensemble des possibilités offertes à un individu est vu comme un don particulier, limité d'une certaine façon par la collectivité, c'est-à-dire par les interdictions légales. Or, on peut décrire toutes les possibilités offertes à un individu d'un point de vue statistique, mais à condition de les isoler, de les séparer du monde dans lequel elles s'insèrent. Ce qui est irréel, puisque chaque individu vit dans un environnement social, dans un système d'actions qui comprend également les autres ${ }^{13}$.

L'interdépendance des individus, l'ensemble des possibilités, le pouvoir, le mode de transaction montrent que le droit de propriété relève d'un consensus social sur ce qui fonde le pouvoir d'un individu sur un autre. Ce consensus tacite est un choix public et il serait naïf de le regarder comme un don de la nature ou l'effet d'une main invisible. En plus, l'explication par l'économie est téléologique et anachronique. Elle est finalisée sur la base d'une fin-valeur d'aujourd'hui: l'économie de marché. Considérant qu'il s'agit du stade final, elle envisage le passé comme une préparation à l'économie du marché capitaliste des biens, des services ainsi que des facteurs les rendant possibles (capital, force-travail, terre). En bref, le passé est reconstruit sur la base des exigences de fondation de la théorie néo-classique contemporaine. Le passé est reconstruit et expliqué uniquement en fonction du présent.

13 Cf. G. Berthoud \& G. Busino, Pratiques sociales et théories. Les discordes des universitaires, Genève, Droz, 1995, pp. 41-94. 
Les limites de ce modèle sont évidentes: trop d'hypothèses simplificatrices (par exemple, le changement institutionnel explique l'évolution des sociétés), un usage immodéré des tests économétriques, l'impossibilité de mettre en relation des concepts invariants avec une réalité en perpétuel changement, une croyance utilitariste dans les mécanismes du marché, dans le profit individuel et dans les droits de propriété comme facteurs essentiels de l'évolution sociale de la société féodale.

L'exemple de la «transition» montre combien grande est la distance séparant les historiens, les économistes et les sociologues, les différences dans leurs réponses, les façons spécifiques d'aborder les mêmes problèmes. Ce désaccord, parfois tumultueux et conflictuel, est d'ordre pratique et théorique. A ce propos, quelques repères essentiels aideront à en comprendre les raisons ${ }^{14}$.

\section{UN DIALOGUE INEXTRICABLE}

Depuis bientôt deux siècles, une querelle a opposé sociologue et historiens à propos de la classification des sciences et de l'épistémologie proprement dite. Depuis l'époque d'Auguste Comte, la question de la place de la sociologie dans la classification des sciences a vainement été débattue par les chercheurs intéressés ${ }^{15}$. Plusieurs d'entre eux ont défendu la subordination à la sociologie de toutes les disciplines ayant pour objet la société; d'autres ont fermement combattu une telle prétention.

Les premiers ont fait de l'histoire une discipline auxiliaire de la sociologie, de même que l'ethnographie et la statistique morale; les seconds (parmi lesquels, par exemple, déjà Numa-Denis Fustel de Coulanges) font de la prétendue science de la société un sous-produit de l'historiographie, une spécialisation quelconque au statut scientifique comparable à celui de la numismatique, de la sigillographie ou de l'héraldique. Charles Seignobos avait coutume de dire que la sociologie est une mode intellectuelle destinée à disparaître comme toutes les modes ${ }^{16}$; Henri Hauser l'accusait de transformer les faits sociaux en formules abstraites.

Entre ces deux impérialismes, les affrontements ont été virulents. L'influence de l'Ecole durkheimienne sur les sciences sociales en France et ailleurs a contribué à répandre la croyance parmi les sociologues, généralement tous positivistes, selon laquelle l'histoire n'est pas une science, mais un art fondé sur la narration chronologique de choses vécues. Les historiens auraient pour tâche de

14 Sur l'utilisation de l'histoire des faits économiques de la part des économistes, voir les textes réunis dans le volume Le vie della storia nell'economia, a cura di Pierluigi Ciocca, Bologna, Il Mulino, 2002. Voir également E. Hobsbawm, Historians and Economists, dans On History, London, Abacus, 2002, pp. 124-163.

15 Cf. G. Weisz, The Emergence of Modern Universities in France, 1863-1914, Princeton, Princeton University Press, 1983, et W. R. Keylor, Academy and Community. The Foundation of the French Academic Culture in Comparative Perspective, 1890-1920, Cambridge, Harvard University Press, 1992.

${ }^{16}$ Cf. M. Rebérioux, Préface à Ch.-V. Langlois et Ch. Seignobos, Introduction aux études historiques, Paris, Kimé, 1992, pp. 7-26; A. Prost, Seignobos revisité, «Vingtième Siècle», n. 43, 1994, pp. 100-118; L. Mucchielli, Une lecture de Langlois et Seignebos, «EspacesTemps Les Cahiers», nn. 59-60-61, 1995, pp. 130-136. Voir aussi O. Dumoulin, Profession historien : un «métier» en crise? 1919-1939, Paris, Ed. de l'EHESS, 1983. 
récolter, vérifier, ordonner des faits individuels épars dans les différents moments de leur devenir, pour ensuite en élaborer un récit dont les finalités sont d'ordre pratique: maintenir en vie, fortifier la mémoire de notre identité sociale et nationale. En revanche, l'analyse, la comparaison, l'interprétation, la recherche des uniformités, des régularités, éventuellement des lois seraient du ressort des sociologues. Dans les sciences de la société, l'explication scientifique ne s'obtiendrait qu'au moyen des rapprochements, des confrontations, de ce que John Stuart Mill dans sa Logique déductive et inductive appelle l'étude des variations concomitantes. Ceci serait irréalisable en historiographie même lorsqu'on étudie les faits mentaux en relation exclusive et complète aux contextes historiques et l'on privilégie le caractère historique des fonctions mentales ${ }^{17}$.

En postulant une différence foncière entre les deux disciplines, en attribuant à la sociologie un domaine très vaste, celui du présent, et à l'histoire le champ du passé, grevé de toutes les servitudes de la documentation, on opposait les visées individualisantes, centrées sur la singularité, de l'histoire à celles généralisantes de la sociologie. L'infériorité épistémologique de l'histoire par rapport à la sociologie était ainsi proclamée ${ }^{18}$.

Cette controverse sur la méthode historique et les sciences sociales a débuté au $\mathrm{XIX}^{\mathrm{e}}$ siècle $^{19}$; elle continue à rester au coeur de la réflexion épistémologique contemporaine ${ }^{20}$. Le dédain pour l'assemblage de faits disparates, pour la reconstruction des événements en leur singularité, cette tâche ne visait, disait-on, qu'à faire passer les faits de l'aléatoire à la cohérence logique, par l'intermédiaire de

17 Sur le sujet, les travaux fondamentaux sont ceux de I. Meyerson, Ecrits 1920-1983. Pour une psychologie historique, Paris, PUF, 1987; Les fonctions psychologiques et les oeuvres. Postface de R. Di Donato, Paris, Albin Michel, 1998; Existe-t-il une nature humaine? Psychologie historique, objective, comparative. Préface de E. Poulat. Introduction de F. Parot, Paris, Institut d'Edition Sanofi-Synthèselabo, 2000.

18 Cf. G. Eisermann, Soziologie und Geschichte, in Handbuch der empirischen Sozialforschung, hrsg von R. König, Stuttgart, Enke, 1972, pp. 601-640; G. Busino, Sociologia e storia. Elementi per un dibattito, Napoli, Guida, 1975 et l'article de G. Noiriel, «Ne tirez pas sur l' historien!». Sur quelques conditions préalables à un «gai savoir» en sciences sociales, «Politix», n. 6, Printemps 1989, pp. 33-39, ainsi que son livre Sur la «crise» de l' histoire, Paris, Belin, 1996. A voir également P. Carrard, Poetics of the new history. French historical discourse from Braudel to Chartier, Baltimore, Johns Hopkins University Press, 1992.

19 Un panorama de ce débat in F. Cantù, Lo storico nella storia, in «Nord e Sud», agosto-settembre 1971, pp. 167-213. La discussion dont on parle dans le texte a été brièvement résumée par F. Braudel, Histoire et sociologie, à présent dans le volume du même, Ecrits sur l'histoire, Paris, Flammarion, 1969, pp. 97-105.

2o Il suffit de renvoyer ici aux livres les plus connus: W. Dray, Laws and Explanations in History, Oxford, Oxford University Press, 1957; A. Danto, Analytical Philosophy of History, Cambridge, Cambridge University Press, 1965; L. Braudy, Narrative Form in History and Fiction: Hume, Fielding \& Gibbon, Princeton, N.J., Princeton University Press, 1973; G. H. von Wright, Explanation and Understanding, London, Routledge and Kegan, 1971; H. White, Metahistory. The historical Imagination in Nineteenth-Century Europe, Baltimore, Johns Hopkins University Press, 1973; Id., Tropics of Discourse. Essays in cultural Criticism, Baltimore, Johns Hopkins University Press, 1978; Id., The Content of the Form: narrative discourse and historical Representation, Baltimore, Johns Hopkins University Press, 1987; R. Aron, Leçons sur l'histoire. Cours du Collège de France. Etablissement du texte, présentation et note par S. Mesure, Paris, de Fallois, 1989. 
causalités singulières. La commisération pour «la poussière des faits » et pour la contrainte littéraire où s'enfermaient les reconstructions historiques allait de pair avec le dédain pour l'imprécision et l'arbitraire des explications dites historiques. Même dans les cas où l'effort portait sur l'établissement de relations causales, comme dans l'œuvre de Paul Lacombe ${ }^{21}$, ou bien s'attachait à reconnaître des déterminismes dans l'événementiel, ou des lois tendancielles dans les processus d'évolution, la supériorité de la sociologie sur l'histoire était affichée.

Dans un article célèbre François Simiand ${ }^{22}$ proclamait qu'il n'y aurait de connaissance que lorsqu'il y a des lois et qu'il n'y a de lois que des phénomènes collectifs. Trois idoles (l'idole politique, l'idole individuelle et l'idole chronologique) obligeraient la tribu des historiens à privilégier les accidents et les actions individuelles, à établir des liens causaux entre un acteur et un acte alors qu'ils sont possibles uniquement entre des phénomènes collectifs du même ordre. Alors que les énoncés scientifiques sont des abstractions, les historiens, selon Simiand, s'occupent d'événements singuliers, des faits vécus; ils prêtent une attention excessive aux petits faits concrets de la vie. Or il n'existe pas de science du singulier, il n'y a de connaissance que lorsqu'il y a des lois et il n'y a de lois que relativement à des phénomènes collectifs. Etant donné que ce sont les événements et les actions individuels qui composent et délimitent le domaine de l'histoire, puisque celle-ci ne parvient pas à s'occuper de phénomènes collectifs, il en découle que l'historiographie n'est pas une science. La mise en relation de faits disparates et la reconstruction d'événements en leur individualité ne peuvent pas, selon les dires de Simiand, faire passer les faits de l'aléatoire à la cohérence logique. En considérant des antécédents choisis au hasard comme la cause du phénomène à expliquer, en établissant des relations entre les intentions et les mobiles d'un acteur et les résultats d'une action sociale, les historiens transgressent la règle selon laquelle il n'y a de rapport causal qu'entre deux phénomènes du même ordre. La cause d'un phénomène est toujours un autre phénomène, antécédent, invariable, non conditionné. La science sociale épure les phénomènes, les débarrasse de leurs dépôts contingents et particuliers; elle étudie des relations stables et régulières, les seules qui possèdent une valeur vraiment significative. L'histoire serait, par ces récits,

\footnotetext{
P. Lacombe, De l' histoire considérée comme science, Paris, Hachette, 1891.

F. Simiand, Méthode historique et science sociale, in «Revue de synthèse historique», Vl, 1903, pp. 1-22 et 129-157 et Méthode historique et sciences sociales. Choix et présentation de M. Cedronio, Paris, Ed. des Archives contemporaines, 1987, ainsi que le recueil d'études François Simiand (1873-1935). Sociologie-Histoire-Economie. Sous la direction de L. Gillard et M. Rosier, Paris, Ed. des Archives contemporaines, 1996. Sur l'empirisme rationaliste et la méthodologie de Simiand, voir les pages de M. Halbwachs, Classes sociales et morphologie. Présentation de V. Karady, Paris, Ed. de Minuit, 1972, pp. 349-389. Pour les prises de positions des sociologues voir: G. Busino, La sociologie sens dessus dessous, Genève, Droz, 1992. Pour les réactions des historiens: Au berceau des «Annales». Le milieu strasbourgeois. L'histoire an France au début du XXe siècle. Sous la direction de Ch.-O.Carbonnel et G. Livet, Toulouse, Institut d'études politiques, 1983. Dans ce volume, lire la contribution de M. Rebérioux, Le débat de 1903: historiens et sociologues, pp. 219-230. Voir également F. Dosse, L' histoire en miettes. Des «Annales» à la «nouvelle histoire», Paris, La Découverte, 1987, ainsi que Ph. Besnard, The epistemological polemic: François Simiand, in Ph. Besnard (ed.), The sociological domain. The Durkheimians and the founding of French Sociology, Cambridge, University Press/Paris, Editions de la MSH, 1983, pp. 248-262.
} 
ces narrations, très proche de la rhétorique et de la fiction ${ }^{23}$. L'objectivité serait étrangère aux historiens. En bref, il leur reproche l'impuissance à construire les objets de recherche, à mettre en valeur les relations spécifiques et universelles qui caractériseraient toute étude à prétention scientifique, la complaisance à l'endroit des travaux descriptifs visant à produire une totalité non construite, faite d'une accumulation inutile de faits disparates ${ }^{24}$.

Le modèle épistémologique à la base d'une telle conception de la science est le même que celui des sciences dures, des sciences de la nature. La reconstruction description du singulier et de l'individuel, du variable et du contingent ne permettrait pas l'élaboration de généralisations, d'expliquer objectivement les causes des faits sociaux, et éventuellement de les prévoir. Seul le collectif, l'invariable, le nécessaire, donnerait lieu à la connaissance scientifique. L'histoire, chronique de la diversité, de la singularité, de tout ce qui n'est pas soumis à la rigide nécessité causale, périodisation qui confère un ordre progressif, cyclique ou alternatif aux événements, l'histoire serait du pur subjectivisme, du savoir pratique pourvoyeur de matériaux bruts pour la construction de la véritable connaissance scientifique.

Tous les historiens refusaient la condition inférieure que Simiand leur octroyait, mais avec des motivations différentes. La conscience collective? Les documents n'en parlent pas. Les réalités sociales? Oui, mais comment les identifier si on fait abstraction de la multiplicité des événements singuliers? Les causes? Comment les déceler entre des antécédents, des motifs et des implications déjà sélectionnés par les documents? Henri Hauser ${ }^{25}$ rappelait avec vigueur qu'une société est une singularité mobile à l'existence permanente, un tout organique, un «Zusammenhang» dont il importe de saisir la spécificité.

Entre 1903 et 1920, sociologues et historiens campèrent résolument sur leurs positions respectives. Malgré l'intérêt pour l'histoire manifesté par Max Weber, et son dialogue ininterrompu avec Eduard Meyer, avec Roscher, Knies, Lamprecht et Treitschke, malgré sa rigoureuse dénonciation du préjugé pour lequel le travail «historique» serait qualitativement différent du travail «scientifique», malgré ses

23 Celle-ci est une question récurrente dans les débats épistémologiques (voir les écrits de Hayden White et de Leo Braudy). On peut lire les remarques les plus pertinentes in A. Momigliano, The Rhetoric of History and the History of Rhetoric. On Hayden White's Tropes (1981), in Settimo contributo alla storia degli studi classici e del mondo antico, Roma, Ed. di storia e letteratura, 1984, pp. 49-59, ed anche in Sui fondamenti della storia antica, Torino, Einaudi, 1984, pp. 465476. Ma sopratutto Tra storia e storicismo, Pisa, Nistri-Lischi, 1985 et Quelle histoire! La «vérité» d'un roman n'est pas la vérité d' un livre d' histoire. Arnoldo Momigliano se fâche, «Le Monde aujourd'hui », dimanche 26-lundi 27 février 1984, p. XIII.

${ }^{24}$ F. Simiand, Méthode historique et sciences sociales. Choix et présentation [par] M. Cedronio, Paris, Ed. des Archives contemporaines, 1987. Sur l'œuvre de Simiand cf. B.-P. Lécuyer, Singularité des faits et vérités statistiques : à partir de la controverse Simiand-Seignobos, in J. Feldman, G. Lagneau, B. Matelon, eds., Moyenne, Centre. Histoire et usages, Paris, Ed. de 1'EHESS, 1991, pp. 275-287; François Simiand (1873-1935). Sociologie-Histoire-Economie. Sous la direction de L. Gillard et M. Rosier, Paris, Ed. des Archives contemporaines, 1996, mais aussi P. Novick, That noble dream. The "objectivity question" and the American historical profession, Cambridge, Cambridge University Press, 1975.

25 H. Hauser, L'enseignement des sciences sociales, Paris, Alcan, 1903 et ce qu'en dit L.-E. Halkin, Initiation à la critique historique, Paris, Colin, 1973, 4éd., pp. 145-150. 
brillants éclaircissements sur la possibilité objective et la causalité adéquate de l'histoire ${ }^{26}$, les rapports entre sociologie et histoire continuèrent à être déterminés et conditionnés par l'empreinte des Français sur le débat, par une incompréhension mutuelle. Et pourtant, les grands sociologues du début du siècle, Durkheim, Pareto et Max Weber, n'ont jamais sous-estimé l'histoire ${ }^{27}$.

Emile Durkheim écrivait en 1888: «Je sais bien que l'historien n'est pas un généralisateur; son rôle tout spécial est, non de trouver des lois, mais de rendre à chaque temps, à chaque peuple, son individualité propre et sa physionomie particulière. Il reste et doit rester dans le particulier. Mais enfin, si particuliers que soient les phénomènes qu'il étudie, il ne se contente pas de les décrire, il les enchaîne les uns aux autres, il en cherche les causes et les conditions. Pour cela il fait des inductions et des hypothèses... $\gg^{28}$. Et une vingtaine d'années plus tard, il précisait: «Il y a, dans l'histoire, du général et du permanent qui peut être traduit en lois; mais il y a aussi du variable, du contingent, qui est imprévisible. L'origine des contingences et l'individu dans toutes ses formes... [voici le domaine de l'histoire]. Le domaine du nécessaire est le domaine de la sociologie $»^{29}$. Histoire, science du contingent, par rapport à la sociologie, science de la nécessité? «L'histoire est le seul instrument dont dispose le sociologue pour résoudre ces sortes de questions [...] En un mot, l'histoire joue, dans l'ordre des réalités sociales, un rôle analogue à celui du microscope dans l'ordre des réalités physiques [...] La sociologie est donc, en grande partie, une sorte d'histoire entendue d'une certaine manière.» D'où la conclusion: «Nous sommes convaincu [...] qu'un jour viendra où l'esprit historique et l'esprit sociologique ne différeront plus que par des nuances $»^{30}$.

Pour Pareto l'histoire est une suite de faits, ressuscités au moyen d'un récit par l'historien, lequel les expose en suivant une méthode chronologique après les avoir individualisés le plus précisément possible. Comme on ne peut faire de contrôles expérimentaux dans les sciences sociales, on doit observer les phénomènes du présent au moyen de la statistique et d'autres techniques. Ces phéno-

26 Cf. C. Calliot-Thélène, Max Weber et l'histoire, Paris, Puf, 1990. Une position semblable a été défendue par Simmel, voir J.-Y. Grenier, La méthode historique de Georg Simmel, in A propos de la «Philosophie de l'argent» de Georg Simmel, Paris, L'Harmattan, 1993, pp. 15-59.

27 Cf. à ce propos H. Stuart Hughes, Consciousness and Society. The Reorientation of European Social Thought, 1890-1930, New York, Vintage Books, 1961, surtout le chapitre II: «The Decade of the 1890's: The Revolt against Positivism». Cf. également la première partie du livre de W.J. Cahnman \& A. Boskoff, Sociology and History. Theory and Research, New York, Free Press, 1964, pp. 19-157, et le livre de T. N. Clark, Prophets and Patrons : the French University and the Emergence of the Social Sciences, Cambridge, Mass., Harvard University Press, 1973. Voir aussi V. Karady, Durkheim, les sciences sociales et l'Université: bilan d'un semi-échec, «Revue française de sociologie»,17, 1976, pp. 267-313; J.-C. Marcel, Le Durkheimisme dans l'entre-deuxguerres, Paris, Puf, 2001, et surtout de Ph. Besnard, Etudes durkheimiennes, Genève, Droz, 2003.

28 E. Durkheim, La science sociale et l'action. Introduction et présentation de J.-Cl. Filloux, Paris, PUF, 1969, p. 107.

29 E. Durkheim, Journal sociologique. Introduction et notes de J. Duvignaud, Paris, PUF, 1969, pp. 674-675

3o E. Durkheim, La science sociale et l'action, cit., pp. 153-157. Sur la conception de l'histoire de Durkheim, cf. R.N. Bellah, Durkheim and History, in W.J. Cahnmann \& A. Boskoff, eds., Sociology and History, cit., pp. 85-103. 
mènes-là demeurent peu nombreux et pas aussi riches d'enseignements que les phénomènes du passé, d'où le recours à: «l'immense valeur des études historiques, lesquelles élargissent considérablement le champ de nos observations.» ${ }^{31}$ Les études historiques intègrent et complètent les études du présent, et grâce à elles nous pouvons effectivement connaître la structure des actions sociales, élaborer une théorie des sociétés. L'histoire est un dépôt d'expériences, une banque de données, des archives ou autres vestiges signifiants, sans lesquels il n'y aurait pas de savoir sociologique ou anthropologique. Que Pareto eût tendance à utiliser l'histoire en tant que collection de faits tirés au hasard d'auteurs et d'époques les plus divers et dont souvent il n'y a pas lieu d'apprécier la valeur représentative, c'est un fait. A ce propos Maurice Halbwachs a observé: «M. Pareto part bien des faits réels, mais il postule l'existence, au sein des faits, de noyaux abstraits, que son analyse l'aide à dégager, et qui deviennent la matière propre de ses constructions. C'est-à-dire qu'il perd tout contact, à partir de ce moment, avec la réalité.» ${ }^{32}$

Ce n'est assurément pas le cas pour Max Weber selon lequel il est impossible de trouver des lois sociologiques, d'établir des types idéaux sans les tirer de l'action historique elle-même. Il y a entre les deux disciplines des problématiques explicitement voisines, une forte solidarité entre l'analyse des événements et l'établissement de propositions générales. La compréhension du fait social exige que l'on recoure à des propositions générales, et celles-ci ne peuvent être démontrées qu'à partir d'analyses et de comparaisons historiques. Les historiens et les sociologues n'utilisent pas les mêmes types sociaux, mais la langue de description du monde historique comporte des caractéristiques qui s'imposent aux uns et aux autres $^{33}$.

Pas de sociologie sans histoire, et pas d'histoire sans sociologie. Werner Sombart et tous les savants qui ont participé à la Methodenstreit ne douteront plus d'une telle affirmation ${ }^{34}$.

Etant donné l'influence prépondérante de l'Ecole durkheimienne sur la sociologie mondiale, le débat sur les sciences de la culture et sur leurs méthodes, sur la sociologie en tant que science et l'histoire en tant qu'art, a subi des conditionnements fâcheux. Les recherches sur l'université française et l'émergence, dans cette institution, de la science sociale, ont révélé la puissance des facteurs de type pratique et montré comment le positivisme scientifique servit de «formule politique », d' «idéologie» dans les stratégies pour la reconnaissance académico-institutionnelle et la légitimité culturelle et professionnelle de la sociologie. Cette

V. Pareto, Scritti sociologici minori, a cura di G. Busino, Torino, Utet, 1980, pp. 242-252.

32 M. Halbwachs, La doctrine sociologique de Vilfredo Pareto, in «Revue d'économie politique», XXII, 1918, pp. 578-585, à présent dans Classes sociales et morphologie. Présentation de V. Karady, Paris, Minuit, 1972, pp. 134-141.

33 M. Weber, Essais sur la théorie de la science, traduits de l'allemand et introduits par J. Freund, Paris, Plon, 1965, pp. 217-323. Une présentation minutieuse des idées de Weber sur l'histoire se trouve in S. Kalberg, Max Weber's Comparative historical Sociology, Cambridge, Polity Press, 1994.

${ }^{34}$ P. Rossi, Sociologia e spiegazione storica, in «Critica storica», I, 1962, pp. 167-180 ainsi que Lo storicismo tedesco contemporaneo, Torino, Einaudi, 1956; Storia e storicismo nella filosofia contemporanea, Milano, Lerici, 1960; Lo storicismo contemporaneo, Torino, Loescher, 1968. 
discipline se devait donc d'affirmer, de revendiquer, d'autovaloriser sa supériorité scientifique afin d'obtenir de la philosophie et de l'histoire un espace académique, culturel et professionnel.

$\mathrm{Au}$ lendemain de cette reconnaissance officielle, la sociologie s'est trouvée en face d'elle une nouvelle génération d'historiens. Les critiques qui s'adressaient aux historiographies de Lavisse, Langlois, Seignobos ou Monod, vont perdre une partie de leur impact. L'historien Marc Bloch, par exemple, ne se limite pas à une exposition de faits chronologiquement ordonnés, enrichis d'anecdotes et de références. Au contraire, dans Les rois thaumaturges (1924), il étudie un miracle, la croyance qui l'entoure et les rituels magiques à travers lesquels parviennent à se manifester le pouvoir du roi à opérer des guérisons ${ }^{35}$. Le même Bloch, dans La société féodale (1939-1940) analyse l'organisation d'une société, sa logique interne; il parvient à nous en restituer le sens, à nous faire comprendre la structure et la complexité de son système de valeurs, à proclamer l'histoire la science des sociétés humaines ${ }^{36}$. Des plus jeunes historiens vont dans la même direction. Louis Vernet, Marcel Granet, Georges Davy étudient la Grèce ancienne, la Chine archaïque, l'Egypte, et réussissent à reconstruire le fonctionnement de systèmes sociaux complexes, à dégager leurs raisons d'être profondes sans recourir à aucune loi, sans laisser échapper ce que ces lointaines sociétés avaient de spécifique et de différent par rapport aux nôtres. La revue les «Annales », créée en 1929, commence à opposer aux abstractions sociologiques l'infinie complexité du social, bien mieux respectée par le concret de l'histoire. Lucien Febvre profitera de toutes les occasions pour rappeler aux sociologues le peu de cas qu'ils font du temps, pour répéter que la vie sociale est plongée dans ce quatrième état de la matière qu'est le temps. Cet argument sera repris par Fernand Braudel dans son écrit sur la «longue durée», où il fera du temps une unité de mesure inscrite dans la réalité37.

Depuis personne ne doutera plus que la perspective historique soit indispensable au travail sociologique. L'anthropologie, en toutes ses tendances évolutionnistes, diffusionnistes et fonctionnalistes, a fait de plus en plus une place de choix à l'histoire ${ }^{38}$. De même, toutes les autres sociologies spéciales ${ }^{39}$. Rarissimes sont

${ }_{35}$ Voir la nouvelle édition de ce livre classique: Les rois thaumaturges. Etude sur le caractère surnaturel attribué à la puissance royale particulièrement en France et en Angleterre. Nouvelle édition. Préface de J. Le Goff, Paris, Gallimard, 1983.

36 M. Bloch, Pour une histoire comparée des sociétés européennes, maintenant in Mélanges historiques. Préface de Ch.-E. Perrin, Paris, SEVPEN, 1963, pp. 16-40. Voir W.H.Sewell jr., Marc Bloch and the logic of comparative history, «History and Theory», 6, 1967, pp. 208-218; A. O. Hill \& B.H.Hill (eds.), Forum Marc Bloch and comparative History, «American Historical Review», LXXXV, 1980, pp. 828-853; D. Romagnoli, La comparazione nell'opera di Marc Bloch : pratica e teoria, in P. Rossi (ed.), La storia comparata. Approcci e prospettive, Milano, Il Saggiatore, 1990, pp. 110-128; H. Atsma \& A. Burguière (éds.), Marc Bloch aujourd'hui. Histoire comparée et sciences sociales, Paris, Ed. EHESS, 1990, pp. 255-236 et pp. 271-278.

37 F. Braudel, Ecrits sur l' histoire, Paris, Flammarion, 1969, mais également Grammaire des civilisations, Paris, Flammarion, 1993 (Ière éd. 1987).

38 A. Deluz-Chiva, Anthropologie, histoire et historiographie, «Revue internationale des sciences sociales », 1965, pp. 624-625, mais également History and Social Anthropology, edited by I.M. Lewis, London, Tavistock, 1968; et History, Sociology and Social Anthropology, «Past and Present », April 1964, p. 102 et suiv. Voir aussi la mise au point de H.R. Trevor-Roper, The Past and the Present of History and Sociology, dans la même revue, February 1969, p. 3 et suiv.

39 Cf. D. Martindale, The Nature and Types of Sociological Theory, Boston, Houghton Mifflin, 
les exceptions: Leopold von Wiese en Allemagne et Benedetto Croce en Italie. Si le premier négligeait l'histoire, le second niait l'existence même de la sociologie en tant que discipline cognitive. Pour le Napolitain il n'y a que l'histoire, la science de toutes les sciences, englobant toutes les manifestations possibles de la vie en société, tout le réel, manifestation suprême de l'esprit absolu: «La seule forme de connaissance est la connaissance historique $»^{40}$. La riposte de Marc Bloch dans son Apologie pour l'histoire était: «Il n'y a [... ] qu'une science des hommes dans le temps et qui sans cesse a besoin d'unir l'étude des morts à celle des vivants ${ }^{41}$, tandis que $\mathrm{Ch}$. Wright Mills constatait que ne pas «poser convenablement les problèmes de notre temps, et notamment celui de la nature des hommes », signifie que nous avons «perdu de vue que l'histoire est le nerf de la science sociale», que nous refusons «d'honorer le principe selon lequel il faut perfectionner une psychologie de l'homme qui soit fondée sur la sociologie et en accord avec l'histoire. S'il est coupé de l'histoire, et s'il n'aborde pas les choses de la psychologie avec un esprit historique, le sociologue n'est pas en mesure de poser convenablement les problèmes qui doivent aujourd'hui orienter ses recherches $»^{42}$.

\section{LES DÉBATS ET LES CONTROVERSES CONTEMPORAINS}

A partir des années 1950 la suprématie des historiens est incontestable, à l'impérialisme sociologique de la première partie du siècle succède l'impérialisme historiographique. La VIe Section de l'Ecole pratique des hautes études, fondée en 1947-1948 par Lucien Febvre, Charles Morazé et Fernand Braudel, regroupera toutes les disciplines autour du noyau dur des historiens. L'histoire se présente désormais comme la reine de toutes les sciences et les sociologues vont se trouver tous sur la défensive. Entre 1956 et 1960, dans une série d'articles percutants, Braudel élimine les dernières résistances. Georges Gurvitch doit le reconnaître à son corps défendant et Georges Friedmann, le sociologue le plus important de ces années-là en France, doit déclarer la mort de l'hégémonisme durkheimien. Dans le discours d'ouverture du IV ${ }^{\text {e }}$ Congrès mondial de sociologie, à Milan, en 1959, il reconnaîtra, en effet, que le savoir sociologique concerne exclusivement les sociétés industrielles. Les connaissances sociologiques pourraient aider ces sociétés à prendre conscience des problèmes suscités par la modernité, à en prévenir les maux, à limiter leur portée. «Autrement dit, face au progrès technique [... ] le sociologue, s’il était mieux équipé et plus souvent consulté, pourrait en contrôler l'introduction, y mieux adapter les collectivités et les individus, aider les

1960, et P. Sorokin, Contemporary sociological Theories, New York, Harper \& Row, 1928, et Sociological Theories of Today, New York, Harper \& Row, 1966.

40 Cf. H. Stuart Hughes, The Evaluation of Sociology in Croce's Theory of History, in W.J. Cahnmann and A. Boskoff, eds, Sociology and History, cit., pp. 128-140.

${ }_{41}$ M. Bloch, Apologie pour l' histoire ou métier d' historien, Paris, Colin, 1961, p. 15. Pour certains prolongements, lire J.-P. Brunet et A. Plessis, Introduction à l'histoire contemporaine, Paris, Colin, 1972.

42 Ch. Wright Mills, L’imagination sociologique, Paris, Maspéro, 1967, p. 151. 
sociétés industrielles à trouver un équilibre qu'elles n'ont pas encore, quelle que soit leur structure, nulle part atteint dans le monde $»^{43}$.

Les sociologues deviennent ainsi les experts des processus de modernisation, ils en décrivent les contradictions, ils en mesurent les rythmes, ils espèrent en préparer et organiser les développements futurs. La recherche empirique, centrée sur des questions sectorielles, sur des événements particuliers, sur des phénomènes singuliers, sur la collecte et la description de données statistiques et d'opinions grâce aux questionnaires, aux entretiens, à l'observation participante, abandonne les débats épistémologiques, néglige les philosophies de la science, oublie les grandes problématiques sur la raison et sur la liberté, et s'intéresse seulement aux réflexions méthodologiques et à l'instrumentation technique de la recherche, à la manière de construire les variables, classifications, typologies, indices, analyse d'observations singulières, construction de systèmes descriptifs, interprétation des relations statistiques, procédures de formalisation, etc.

Les abus engendrés par cette habitude ont été dénoncés avec une verve exceptionnelle par Pitirim Sorokin et par Charles Wright Mills ${ }^{44}$. Ce dernier parlera de grande théorisation et d'empirisme abstrait impuissant à nous apprendre quoi que ce soit de valable sur l'homme et sur la société, à nous faire « comprendre la biographie et l'histoire et les rapports entre l'une et l'autre en une variété de structures sociales ».

Les sociologues, devenus conseillers du Prince, concentrent leurs efforts sur le présent, voire sur l'actualité la plus immédiate. Les historiens, au contraire, consolident leur présence tant dans les secteurs de recherche sur le présent que dans celui sur le passé et ne perdent aucune occasion pour étendre leurs interventions. Ils s'emparent de toutes les techniques mises au point dans les recherches sociographiques et s'approprient ainsi des secteurs de la connaissance qu'ils n'avaient jusque là pas explorés. L'histoire des hiérarchies sociales, des processus sociaux de construction, de leurs évolutions, permet de faire comprendre la dynamique des luttes sociales sans recourir à des formes rigides de causalité. La microhistoire et l' «Alltagsgeschichte ${ }^{45}$ permettent également de se soustraire aux réalismes, substantivismes et réductionnismes courants, habituels dans l'ancienne historiographie économique et sociale, d'échapper au relativisme méthodologique au nom de la rationalité limitée. Elles parviennent aisément à faire le lien entre le passé et le présent, à montrer le rapport continuité / discontinuité dans les cycles de transition, à reconfigurer le temps et les rapports local/total, particulier/universel. Le changement d'échelle fait surgir des modèles de compréhension plus opératoires, mieux des agrégats statistiques, et permet de prendre en compte les marges que dansa les conduites personnelles ont été laissées aux acteurs par les formes de domination ${ }^{46}$.

43 G. Friedmann, La puissance et la sagesse, Paris, Gallimard, 1970.

44 P. A. Sorokin, Sociological Theories of Today, New York, Harper, 1966; Tendances et déboires de la sociologie américaine. Préface de G. Gurvitch, Paris, Aubier, 1959. Et C. Wright Mills, The Sociological Imagination, Oxford, Oxford University Press, 1959, spéc. les chapitres de II à VI.

45 A. Banti, «Storie et Micro-storie»: l'histoire sociale contemporaine en Italie (1972-1989), «Genèse», 3, mars 1991, pp. -, et C. Conrad \& M. Kessel (Hgb.), Geschichte schreiben in der Postmodern, Stuttgart, Reclam, 1994.

46 G. Levi, On Microhistory, in P. Burke, New Perspectives in Historical Writing, Oxford, Polity Press, 1991, pp. 93-113 et E. Grendi, Microanalisi e storia sociale, «Quaderni storici», n. 33, 
L'affaire semblait être classée pour de bon lorsque Claude Lévi-Strauss relança le débat en montrant «que la différence fondamentale entre les deux [disciplines] n'est ni d'objet, ni de but, ni de méthode; mais qu'ayant le même objet, qui est la vie sociale; le même but, qui est une meilleure intelligence de l'homme; et une méthode où varie seulement le dosage des procédés de recherche; elles se distinguent surtout par le choix de perspectives complémentaires: l'histoire organisant ses données par rapport aux expressions conscientes, l'ethnologie [ou la sociologie] par rapport aux conditions inconscientes, de la vie sociale $\gg^{47}$.

Les historiens se sentirent touchés par une telle division des tâches. N'avaientils pas essayé de récupérer le non événementiel dans l'histoire, mis l'accent sur les conditions latentes de la vie sociale, d'expliquer les sociétés par leur système de fonctionnement, par leurs modes de production manifestes et latents? Leur amour-propre ne fut pas satisfait lorsque, dans Du miel aux cendres, Lévi-Strauss écrira: «En affirmant ses prétentions aussi résolument qu'elle l'a fait dans ce livre, l'analyse structurale ne récuse donc pas l'histoire. Bien au contraire, elle lui concède une place de premier plan: celle qui revient de droit à la contingence irréductible sans laquelle on ne pourrait même pas concevoir la nécessité ${ }^{48}$.

Fernand Braudel essaya de renverser le problème en faisant valoir que la seule chose essentielle est la durée, la longue durée: «Ce n'est pas à l'histoire qu'en ont, finalement et inconsciemment, les sociologues, mais au temps de l'histoire, cette réalité qui reste violente, même si l'on cherche à l'aménager, à la diversifier. Cette contrainte à laquelle l'historien n'échappe jamais, les sociologues, eux, y échappent presque toujours: ils s'évadent, ou dans l'instant, toujours actuel, comme suspendu au-dessus du temps, ou dans les phénomènes clé répétition qui ne sont d'aucun âge; donc par une démarche opposée de l'esprit, qui les cantonne soit dans l'événementiel le plus strict, soit dans la durée la plus longue. Cette évasion est-elle licite? Là est le vrai débat $[\ldots] »^{49}$. Temps longs? Plutôt, temps réversible et cumulatif, changeant d'après les besoins et les nécessités de chaque culture. Temps en tant qu'élément des transformations, en tant que rythme marquant les passages d'une équilibration à l'autre, d'un stade à l'autre. En ce sens le temps est englobé en tout, même dans l'événement, qu'il a contribué à mûrir; même dans le non-événementiel dont il a ponctué les équilibrations, dont il a scandé le possible et le réel. Temps courts et temps longs? Cela dépend de la conception que chaque culture s'en faisait. Il a été noté que: "Nous étudions l'histoire [...] pour connaître le monde dans lequel nous vivons, la réalité sociale qui nous entoure, en tant que ce monde et que cette réalité se composent d'éléments qui sont anciens, ou qui ont eu des précédents, et ne se laissent comprendre que

1972, pp. 506-520; J. Schlumbohm. Ed., Mikrogeschicte/Makrogeschichte Komplementar oder Inkommensurabel?, Göttingen, Wallstein, 1998. Voir aussi G. Busino, Causalisme, symétrie et réflexivité. Une lecture des travaux de Carlo Ginzburg, in L'acteur et ses raisons. Mélanges en l' honneur de Raymond Boudon, Paris, Puf, 2000, pp. 25-42.

47 Cl. Lévi-Strauss, Anthropologie structurale, Paris, Plon, 1958, p. 25.

48 Cl. Lévi-Strauss, Mythologiques. ** Du miel aux cendres, Paris, Plon, 1966, p. 408. Sur toute la question, cf. Orte des wilden Denkens. Zur Anthropologie von Claude Lévi-Strauss, hrsg. von W. Lepenies und H. Henning Ritter, Frankfurt, Suhrkamp, 1970.

49 F. Braudel, Ecrits sur l' histoire, cit., spéc. la deuxième partie. 
par leur genèse. L'objet de l'histoire est donc, avant tout, ce qui change, ce qui se métamorphose - ou s'est métamorphosé [...] L'histoire analysera des états de choses et des évolutions [...] Elle en connaîtra les intentions et en suivra attentivement les résultats $»^{50}$.

Admis que la durée caractérise l'histoire, il reste toujours à faire l'histoire des événements, des manifestations visibles et en partie contingentes, l'histoire des dynamismes sous-jacents, des processus d'élaboration et de développement. Chaque phénomène est complexe, incorpore le temps, et il est redevable à des processus d'intégration croissante, à une organisation progressive ou structuration $^{51}$.

A partir de 1968 une crise profonde secoue toutes les certitudes de la sociologie. Les sociologues commencent à douter même de leur propre rôle social. La crise économique internationale, dès les années 1973-1974, accélère la chute des illusions et chasse les mirages ${ }^{52}$. Les modèles formels destinés à schématiser le réel, à rendre compte des processus de modernisation, ne parviennent pas à expliquer pourquoi les changements sociaux annoncés se heurtent à des refus, à des résistances, à des rejets violents. Les préjugés nomologiques, structuralistes et ontologiques n'aident pas à isoler dans la masse des données historiques un phénomène macrosocial afin d'en connaître la raison d'être, de mettre en lumière les lois générales de type probabiliste régissant le dit phénomène, d'en rendre compte dans sa singularité mais de l'interpréter comme la réalisation singulière de structures plus générales ${ }^{53}$.

so Aufour, Histoire politique et psychologie historique, Genève, Droz, 1966, p. 25. Ces idées ont été reprises récemment dans l'article Comment on écrit l'histoire et comment on la pense, in «Revue européenne des sciences sociales», X, 1972, n. 27, pp. 171-177. Cf. également E.H. Tumas, Economic history and the social sciences. Problems of methodology, Berkeley-Los Angeles, University of California Press, 1971, spéc. les pp. 293-309.

51 Cf. J. Piaget, Le structuralisme, Paris, PUF, 1968. Cf. également le livre d'A. Dupront, L'acculturazione. Storia e scienze umane, Prefazione e traduzione di C. Vivanti, Torino, Einaudi, 1966, mais surtout Ph. Abrams, Historical Sociology, Near Shepton Mallet, Somerset, Open Books, 1982.

52 Cf. l'article de M. Pollak, L'historien et le sociologue: le tournant des années 1980, in Ecrire l' histoire du temps présent. En hommage à François Bedarida, Paris, CNRS, 1993, pp. 329-359. Sur la nouvelle génération d'historiens post-durkheimiens, voir Ph. Besnard, Y. Goudineau et J. Ravel, in Historiens et Sociologues aujourd' hui. Journée d'études annuelles de la Société française de Sociologie. Université de Lille I, 14-15 juin 1984, Paris, CNRS, 1986; C. Delacroix, La falaise et le rivage. Histoire d' un 'tournant critique', «EspacesTemps. Les Cahiers», nn. 59-6061, 1995, pp. 86-111.

53 R. Boudon, La place du désordre. Critique des théories du changement social, Paris, PUF, 1984; Les problèmes de la philosophie de l' histoire de Simmel, in Historien et sociologues aujourd' hui, op. cit., pp. 179-183, ainsi que dans Individualisme et holisme: un débat méthodologique fondamental, in H. Mendras \& M. Verret (eds.), Les champs de la sociologie française, Paris, Colin, 1988, pp. 31-45. Dans ce même volume, voir l'article de M. Aymard, Histoire et sociologie, op. cit., pp. 221-232. Pour un résumé de la pensée du sociologue français sur les rapports sociologiehistoire, voire R. Boudon \& F. Bourricaud, Histoire et sociologie, dans Dictionnaire critique de la sociologie, Paris, Quadrige/PUF, 2000, pp. 279-287. R. Boudon dans Raisons, bonnes raisons, Paris, Puf, 2003, spéc. p. 145 et suiv., revient sur cette question des rapports entre l'histoire et la sociologie et affirme que les historiens s'intéressent plutôt à des sujets relevant de l'interprétation alors que les sociologues s'intéresseraient plutôt au sujet relevant de l'explication. 
Pendant ces années-là, Michel Foucault s'acharne à dénoncer les absurdes prétentions des sociologues, leur positivisme obtus, leur culte insensé de la modernité, la vanité de leur savoir emprisonné dans un système discursif produit par des lois et des déterminismes inconscients, qui n'ont rien à voir avec le progrès de la raison et avec la liberté de l'homme. L'objet des catégories d'analyse de la réalité sociale est le fruit d'opérations dominantes d'objectivation à un moment historique déterminé. Les changements observables dans les sciences humaines ne constituent pas un progrès. Ils ne sont qu'une nouvelle variante du mode d'organisation et d'appropriation des comportements sociaux. Le changement dans les discours - et pour Foucault le discours est une succession d'affirmations, de pratiques, des schèmes classificatoires et d'objets d'analyse lesquels, bien que disparates et contradictoires, contiennent l'ensemble des règles qui gouvernent le fonctionnement discursif tout entier -, le passage d'un ensemble de discours (épistémè) à un autre ensemble ne constituent pas un progrès de la rationalité, ne sont même pas produits ou déterminés par les conditions matérielles dominantes à une époque donnée. Chaque société et chaque époque ont des types de discours considérés comme vrais, mais qui sont en réalité produits par les multiples formes de construction en vigueur. En somme, l'arbitraire, le contingent, le non rationnel sont les caractéristiques majeures des comportements humains. Les catégories analytiques et les techniques de la sociologie (comme du reste de toutes les sciences sociales et humaines) n'ont d'autre utilité que de dissimuler les rapports de pouvoir, à fragmenter la réalité en une myriade d'entités séparées les unes des autres. Et c'est ainsi que les systèmes de domination se perpétueraient ${ }^{54}$.

De son côté, Paul Ricoeur a attiré l'attention des « Social Scientists » sur la nécessité d'étudier l'expérience temporelle à partir de ses mises en récit dans la fiction et l'histoire, tandis que Pierre Bourdieu les a incités à tenir les «différentes manières de temporaliser»comme une propriété sociale distinctive qui oppose, aux deux extrêmes du champ social, ceux qui sont maîtres de leur temps et celui des autres, et les «hommes sans avenir» qui vivent le «non-temps d'une vie où il ne se passe rien et où il n'y a rien à attendre »; Reinhart Koselleck, quant à lui, fait de la tension entre les horizons d'attente et les expériences vécues la clef des transformations du rapport des sociétés à la temporalité55. Pour sa part François Chazel proclame qu' «Entre une histoire interprétative, dégagée des excès de l'histoire pseudo-systématique et soucieuse de dépasser les fausses évidences de la chronique, et une sociologie ouverte sur la comparaison, engagée dans la production d'outils conceptuels et davantage préoccupée de modèles que de lois pourrait se développer une collaboration féconde, plus suivie qu'elle ne l'a généralement été au cours du siècle passé »56.

54 J.-G. Merquior, Foucault, London, Fontana Paperbacks, 1985; R. Rorty, Méthode, science sociale et espoir social, «Critique», XLII, n. 471-472, août-septembre 1986, pp. 873-897; M. Poster, Foucault, le présent et l' histoire, in Michel Foucault philosophe. Rencontre internationale, Paris 9, 10, 11 janvier 1988, Paris, Seuil, 1989, pp. 354-371. Dans le volume édité par M. Perrot, L'impossible prison, Paris, Ed. du Seuil, 1980, outre plusieurs études historiques sur les prisons, sont rassemblés les éléments principaux de la discussion entre Foucault et les historiens.

5s Toutes ces questions sont magistralement traitées par F. Hartog, Régimes d' historicité. Présentisme et expérience du temps, Paris, Ed. du Seuil, 2003.

56 F. Chazel, Sur quelles bases établir des relations stables entre historiens et sociologues?, in Qu'est-ce qu'on ne sait pas en histoire, publié sous la direction d'Y. Beauvois et C. Blondel, Bordeaux, Presses Universitaires du Septentrion, 1998, pp. 117-129. 
Face à ces dangers de liquidation culturelle et académique de la sociologie, trois stratégies ${ }^{57}$ ont été adoptées: (a) la revendication «mise à jour» de la spécificité et de la scientificité de la discipline et son absolue égalité épistémologique par rapport à l'histoire; (b) la création d'une sociologie historique, surtout dans les pays anglo-saxons; (c) la re-fondation d'une nouvelle sociologie sur la base d'une critique sévère des formations sociales finalistes et de la construction d'un paradigme méthodologique de type individualiste.

La première stratégie, adoptée par Pierre Bourdieu ${ }^{58}$ et Jean Claude Passeron $^{59}$, de façon plus nuancée, reformule, en tenant compte de nombreux acquis historiographiques et des thèses de Marx et de Weber à la fois, le vieux paradigme durkheimien.

Bourdieu conteste les critiques des historiens et polémique contre leur propension à considérer que la recherche historique est recherche de la genèse, des origines, des responsabilités, que le sens de l'histoire est contenu dans les actions historiques. C'est ceci qui introduit, selon Bourdieu, une vision machiavélique et téléologique de l'histoire. Les institutions et les ensembles collectifs (Etat, Eglise, Famille, Nation, Groupe, Communauté, Classe, etc.) ne sont pas des sujets historiques capables d'élaborer leurs propres finalités. La raison d'être de ces institutions et celle de leurs effets sociaux ne se trouve pas dans la volonté des individus ou des groupes, mais dans le camp des forces antagonistes ou complémentaires. L'action sociale est le produit d'une rencontre entre deux états de l'histoire: l'histoire à l'état objectivé (machines, monuments, tableaux, presse, livres, théories, coutumes, etc.) et l'histoire à l'état incorporé (dispositions, habitus). Ce qui a été s'inscrit dans les choses et dans les corps et augmente la part d'irréversible que l'on constate dans le présent. La sociologie, en objectivant «ce qu'il y a d'impensé social, c'est-à-dire d'histoire oubliée» dans le présent, détruit les impostures et les illusions, révèle le poids du passé sur le présent et les parts respectives de l'histoire réifiée et de l'histoire incorporée. Puisque le passé est incorporé en nous et objectivé dans le présent, le sociologue est porté à s'occuper d'études du présent sans se sentir tenu à analyser la genèse et l'évolution, et donc à traiter le temps à la façon des historiens. Néanmoins, il ne s'agit pas d'une limite absolue. La sociologie peut étudier n'importe quel domaine à n'importe quelle époque, à condition de ne pas renoncer à l'emploi de schèmes d'interrogation et d'explication universels. Pour Bourdieu il y a aussi une autre différence structurelle entre l'histoire et la sociologie. Alors que les objets historiques sont toujours à distance des historiens, et que les intérêts de ces derniers n'y sont jamais directement

57 J.-C. Passeron m'a fait observer qu'il s'agit plutôt d'une description épistémologique de la sociologie telle qu'elle se pratique en fait chez tout sociologue et non d'une stratégie. La question mériterait un plus ample approfondissement.

58 Cf. P. Bourdieu, Le mort saisit le vif. Les relations entre l' histoire réifiée et l' histoire incorporée, «Actes de la recherche en sciences sociales», n. 32-33, avril-juin 1980, pp. 3-14.

59 Cf. J.-C. Passeron, Histoire et sociologie: identité sociale et identité logique d' une discipline, in Historiens et sociologues aujourd' hui, op. cit., pp. 195-208,; Hegel ou le passager clandestin. La reproduction sociale et l' histoire, «Esprit», juin 1986, pp. 63-81; Le raisonnement sociologique. L'espace non-poppérien du raisonnement naturel, Paris, Nathan, 1991; L'enseignement, lieu de rencontre entre historiens et sociologues, «Sociétés contemporaines», n. 1, mai 1990, pp. 7-45; Anthropologie et Sociologie, «Raison présente », n. 108, $4^{\text {ème }}$ trimestre 1993, pp. 1-44. 
impliqués, en sociologie les rapports entre le sujet connaissant et l'objet à connaître, entre le subjectivisme et l'objectivisme, entre la distance et l'implication, sont très forts.

Jean-Claude Passeron modifiera de façon substantielle cette conception, puisqu'il doute plus radicalement encore de l'existence d'une loi du changement historique ou de «lois » particulières qui, en chaque sciences sociales, gouverneraient les modalités de passage de l'équilibre synchronique à l'évolution diachronique. Pour lui, le recours à des concepts et à des « régularités » reste au principe des généralités que peut formuler un discours sociologique, tant du moins que le sociologue ne se méprend pas sur le sens de la logique idéal-typique dont Weber avait révélé l'omniprésence dans les sciences historiques. Le concept de reproduction sociale, par exemple, reste utile à la description diachronique, tant que les structures d'un système d'interdépendance demeurent stables, à condition donc, de ne jamais confondre - comme le faisait Marx et, aujourd'hui encore, bien des sociologues pratiquant un déterminisme unilinéaire - la logique opérationnelle d'une construction interprétative avec la nécessité historique elle-même. Sociologie et histoire sont indiscernables du point de vue épistémologique. La logique des définitions inscrite dans leur langue de description du monde historique implique, chez l'une comme chez l'autre, l'impossibilité sémantique d'un paradigme entièrement unifié. Leurs concepts typologiques, leurs méthodes d'analyse des «cas» ne peuvent pas être «désindexés» des «contextes» où surviennent leurs événements.

Passeron s'est donné pour tâche, après Le métier de sociologue et La reproduction (derniers ouvrages co-écrits avec Bourdieu, 1968 et 1972), d'explorer et de décrire cette convergence épistémologique entre histoire et sociologie en même temps que leurs différences dans le «dosage» des méthodes; et, plus généralement, d'expliquer pourquoi les sciences sociales ne sont vraiment «sociales » que tant qu'elles restent, en leur fond, des sciences historiques. L'histoire construit ses «faits» en référant ses interprétations au contexte spatio-temporel des phénomènes observés. La sociologie, de son côté, veut tirer parti, pour fonder ses assertions historiques, des corrélations, des parallélismes ou des contrastes dont la constance approximative est dotée d'une certaine durée. Ses preuves proviennent essentiellement de comparaisons entre données observées et, dans le meilleur des cas, mesurées, non certes «toutes choses étant égales par ailleurs » mais dans des contextes analogues. A la sociologie appartient en propre l'établissement de « régularités » qui dotent un récit historique de ses cadres de généralité; à l'histoire la description des changements et de leurs allures, régressives, progressives ou cycliques, qui donnent un sens aux constats parcellaires du sociologue. L'histoire est la discipline qui illustre le mieux la démarche consistant à ancrer, en chacun de ses actes d'explication, ses synthèses interprétatives dans le contexte spatiotemporel des phénomènes observés. La sociologie illustre, pour atteindre au même but, une démarche qui fonde sa construction de preuves sur un «va-et-vient» méthodologique entre le raisonnement historique et le raisonnement expérimental. Histoire et Sociologie ne sont finalement distinctes qu'au plan de leurs dosages méthodologiques. Cela suffit pour leur conférer une identité scientifique distincte. Lorsqu'elle se fait «sociologie historique», la sociologie reste, pour toutes ses méthodes, de la sociologie, de même que l'«histoire sociale» reste de l'histoire. Confondre totalement leurs méthodes aboutirait à affaiblir leurs forces respectives de compréhension et d'explication, en les spécialisant par leur seul rapport à 
l'actualité: pour l'histoire ce serait se plier sur une interprétation linéaire des continuités de longue durée; et pour la sociologie se confiner dans une analyse du présent des individus ou des groupes, dont le principe actif ne peut plus être alors incarné que dans un habitus qui n'intérioriserait jamais une contrainte objective par laquelle les systèmes de domination et de contrôle social se reproduisent monotonement. En réalité, les deux disciplines sont vouées à échanger continûment leurs résultats, à féconder leurs intelligibilités parentes, travaillant sur un même terrain de prospection des données et partageant une même définition de l'historicité des actions sociales. En somme, les descriptions de la sociologie et de l'histoire que dessinent les analyses de Passeron n'imposent pas une «ligne » épistémologique qui leur conseillerait de se remodeler ou de rompre avec leur passé pour préserver ou acquérir leur pleine spécificité; il formule simplement un bilan: moins un pronostic qu'un diagnostic de leur forme de scientificité. Tous les arguments qu'il donne de la spécificité de leur régime scientifique sont fondés sur une description factuelle des pratiques de ces deux disciplines: ce n'est donc pas une stratégie de re-fondation qu'il propose à ces disciplines; tout au plus une règle de lucidité épistémologique sur la portée assertorique de leurs actes et travaux scientifiques. Pour conclure, entre ces disciplines la différence provient du style méthodologique. Les règles du discours pour faire la preuve, pour faire le sens, pour produire l'intelligibilité tiennent au fait que le récit historique «est contraint, par l'abondance et le rôle argumentatif de ses 'déictiques', à construire un autre rapport probatoire entre la généralité et la singularité».

Mais si les sciences sociales sont des sciences historiques, si tous les objets sociaux sont historiques, les théories sociologiques sont alors des théories interprétatives, des langages de description du monde historique, fondés sur l'observation, la comparaison, la typologie. Pour cette raison «nos raisonnements s'inscrivent donc dans le cadre d'une science empirique, dont toute la scientificité tient à ce qu'elle est capable de se soumettre aux contraintes d'un espace logique, celui de l'enquête, en tant que celle-ci organise de manière stable le sens des assertions ».

Encore pour cette raison «en anthropologie et sociologie, les seuls modèles utilisables sont des modèles interprétatifs. Et seule la rigueur de la méthode dont on se sert pour construire l'interprétation décide de leur caractère scientifique ou non ». Dès lors les sciences sociales produisent des connaissances d'un autre ordre que celles du sens commun. L' «intelligibilité» produite par les sciences sociales est comparative, ne préexiste pas dans les données empiriques. Le statut scientifique des sciences sociales se fonde sur le raisonnement naturel, sur une «autre logique », la logique non démonstrative, la logique argumentative.

La deuxième stratégie de défense adoptée par les sociologues consiste à transformer la sociologie en sociologie historique. L'ouvrage de Norbert Elias Ueber den Prozess der Zivilisation, paru en 1939 mais réellement connu au début des années ' 70 , aura un rôle déterminant dans les tentatives d'ancrer la recherche sociologique dans les manifestations de l'historicité60.

${ }^{6}$ Cf. Materialen zu Norbert Elias «Zivisationstheorie». Herausgegeben von P. Gleichmann, J. Goudsblom und H. Korte, Framkfurt a. M., Suhrkamp, 1977, ainsi que A. Bogner, Zivilisation und Rationalisierung: die Zivilisationstheorien Max Weber, Norbert Elias un die Frankfurter Schule im Vergleich, Opladen, Westdeutscher Verlag, 1989. Lire également F. Dosse, L'empire du sens. L'humanisation des sciences humaines, Paris, La Découverte, 1995, pp. 108-116. 
Elias affirme que l'histoire met l'accent sur les événements et les personnalités alors que la sociologie dévoile les systèmes d'interdépendance liant l'ensemble de ces facteurs et en les organisant en une configuration singulière et spécifique. A l'intérieur de telles formations les individus disposent d'une marge de liberté, mais les interdépendances qui les lient aux autres limitent leur liberté de choix. Il y a une articulation constante entre la transformation des sensibilités humaines, des comportements sociaux, et la construction de l'Etat; entre la formation de l'Etat et la différenciation des fonctions sociales, entre les processus de civilisation, la structure du pouvoir, les relations sociales et les comportements individuels et les normes esthétiques. En d'autres termes, l'interdépendance existant entre les différents niveaux de réalité et la complexité des voies à travers lesquelles s'effectuent les échanges réciproques, est un processus complexes. Les mécanismes d'intériorisation des principes rationnels et l'incorporation des normes de conduite permettent d'imbriquer l'individuel avec le collectif, le mental avec le comportemental, la psychologie avec la sociologie, la sociologie avec l'histoire.

L'histoire nous parle de l'unique, du singulier, de l'apparent, du conscient, de ce qui change; la sociologie nous révèle le latent, le pluriel, le constant, l'inconscient. Les déterminations sont réciproques et les échanges continus. Les formations sociales changent parce que les hommes changent; et les hommes changent parce que les systèmes d'interdépendance se modifient constamment, de manière endogène et de manière exogène. La Société de Cour est l'exemple typique de cette imbrication analytique entre la genèse des comportements rapportés aux contextes singuliers, leur fonctionnement et leur évolution. Les volumes de Über den Prozess der Zivilisation, de Über die Zeit et Engagement und Distanzierung, montrent la finesse et la plasticité de la construction théorique d'Elias. Ses thèses ont suscité une nuée de vocations. Peter Burke, par exemple, propose que l'histoire et la sociologie unifient leurs programmes de travail, qu'elles donnent lieu à une synthèse méthodologico-disciplinaire. L'histoire fournirait les matériaux, les reconstructions, la compréhension des phénomènes, la sociologie les modèles interprétatifs. La sociologie deviendrait ainsi la méthodologie de l'historiographie $^{61}$. Un autre Anglais, Philip Abrams, lui aussi convaincu de la nécessité d'une forte intégration entre la sociologie et l'histoire (toutes les deux se proposent d'expliquer l'agir humain) a montré, à travers l'étude d'une série de questions (l'anomie, la formation des classes, la lutte de classe, la société industrielle, la formation des Etats, les générations, les monstres et les héros humains, etc.) que les deux disciplines «cherchent à comprendre le problème de l'action humaine et toutes deux cherchent à le résoudre en faisant référence aux processus de structuration sociale $»^{62}$. Les différences entre l'histoire et la sociologie dépendraient de la manière de reconstruire la réalité sociale, de la façon d'argumenter cette reconstruction et ensuite de la faire valoir. Il s'agirait de différences argumentatives, rhétoriques.

61 P. Burke, Sociology and History, London, Allen \& Unwin, 1980.

62 J.H. Abrams, Historical Sociology, Somerset, Open Books, 1982, mais aussi son The Origins and Growth of Sociology, Harmondsworth, Penguin Books, 1973. Du même History, Sociology, Historical Sociology, "Past and Present", 1980, n.87, pp. 3-16. 
Un exemple plus convaincant de l'enrichissement mutuel entre histoire et sociologie nous est fourni par les travaux d'Immanuel Wallerstein sur le capitalisme historique ainsi que ceux de Charles Tilly, avant tout par son livre sur la révolution et contre-révolution en Vendée et dans la France de l'Ouest, paru en anglais en 1964 et dans une nouvelle édition, en français, en 197063.

L'analyse sociologique du matériel historique a renouvelé complètement la problématique: l'Ouest ne s'est pas révolté pour défendre le Trône, mais à cause des préjugés parisiens à l'égard de certaines régions. Après l'éclatement de la révolte, les nobles ont tout fait pour la récupérer et lui donner d'autres objectifs. L'idée que les Vendéens se soient révoltés pour défendre l'Ancien Régime est une idée fausse, fruit de rationalisations rétrospectives de la part des descendants de ceux qui subirent d'horribles répressions, et de la part des historiens qui les reconstituèrent. Et les sociologues d'aujourd'hui étudiant le comportement électoral de la Vendée sont eux aussi tombés dans ce piège.

La nécessité d'ouvrir la sociologie à l'histoire est argumentée ainsi : l'histoire reste le seul moyen pour neutraliser l'ethnocentrisme et les partis pris idéologiques dissimulés par les sociologues. De plus, elle a permis de revisiter avec des perspectives et des matériaux nouveaux, provenant d'époques et de lieux différents, d'anciennes problématiques, par exemple, les rapports ethniques dans les villes américaines, l'émergence de l'Etat Providence et des politiques sociales, les révoltes des étudiants et des minorités ethniques, le développement des professions libérales, la formation et le déclin des idéologies, les fonctions des systèmes culturels. L'analyse des phénomènes idéologiques dans le temps et dans l'espace a permis de comprendre pourquoi les idéologies peuvent ne pas se rapporter aux intérêts et aux stratégies d'un collectif déterminé (le patronat, la classe, la bourgeoisie, le clergé, etc.); elle a révélé en outre que les idéologies sont avant tout des formes discursives, cognitives, émotives, impersonnelles, anonymes, propres à des groupes entiers. Les idéologies justifient, rationalisent, garantissent les croyances, les expériences, les rendent utilisables pour l'action et pour la vie de tous les jours. Pas nécessairement propagées par un groupe précis, exerçant une influence indépendamment des attentes et des intérêts des acteurs sociaux, les idéologies possèdent une dynamique symbolique relativement autonome, dotée d'une temporalité et d'effets propres.

Le courant de sociologie historique réserve une attention particulière également à la localisation spatiale des processus étudiés, en reliant les actions significatives aux contextes structurels locaux, dont les variations et les spécificités sont ensuite valorisées. C'est non seulement le temps, mais aussi les lieux qui sont ainsi incorporés à l'analyse sociologique, auparavant indifférente aux localisations spatiales et temporelles. Si tous les systèmes sociaux réels sont transformés par des acteurs historiques datés et situés; si les attitudes, les pratiques, les représentations humaines sont la résultante de conditions culturelles précises, variables dans le temps et dans l'espace; si chaque société produit, de manière inéluctable, des tensions, des contradictions et des conflits, qui débouchent ensuite, selon des

63 C. Tilly, L' histoire à venir, «Politix», n. 6, Printemps 1989, pp. 25-32. Lire aussi le numéro monographique intitulé Sociologie historique de la «Revue internationale des sciences sociales», n. 133, août 1992, et T. Skocpol ed., Vision and Method in Historical Sociology, Cambridge, Cambridge University Press, 1984. 
rythmes temporels imprévisibles, sur des changements structurels qui sont toujours différents de ceux poursuivis intentionnellement et délibérément par les acteurs; si les conséquences systémiques, volontaires ou involontaires, dépendent non seulement de la constellation des objectifs et des ressources des parties en lice, mais également d'une articulation qui leur est propre, - alors les acteurs sociaux sont capables de réflexivité puisqu'ils adaptent leurs actions et réactions, conforment leurs habitudes, leurs désirs, leurs besoins et même leurs visions du monde social, ainsi que les rationalisations idéologiques qui leur sont inhérentes, aux transformations objectives.

Que cela soit suffisant pour résoudre le problème des rapports de la sociologie avec l'histoire, c'est douteux ${ }^{64}$.

Une théorie de l'action rationnelle a été proposée à la place par les tenants de la troisième stratégie de défense visant à sortir la sociologie de la crise où elle se débat. C'est l'approche dénommé de l'action rationnelle (Rational Action Theory ou RAT), subdivisée en diverses sous-approches: Choix rationnels, Action collective, Microfondements, Individualisme méthodologique. La RAT prend le contrepied du fonctionnalisme dominant en sociologie, et surtout des tendances interprétatives ou cognitives, et plus particulièrement de l'interactionnisme symbolique, de l'ethnométhodologie, de la sociologie phénoménologique, du behaviorisme et de la théorie économique de l'échange. Certains théoriciens de la $R A T$ postulent que tous les phénomènes sociaux sont des phénomènes historiques et tous sont le produit ou l'expression de phénomènes psychiques. Ces derniers dépendent des circonstances dans lesquelles les acteurs se sont trouvés dans le passé et dans un lieu donné, ainsi que de la situation et de l'environnement où ils se trouvent dans le présent. L'explication suppose la connaissance exacte de ces facteurs et des médiations qu'ils ont effectuées et effectuent encore. Tous les phénomènes sociaux sont toujours les résultantes d'actions individuelles insérées dans des contextes structurels, ils sont le produit d'acteurs historiques, avec un passé qui façonne le présent. Agrégation d'actions dictées par des motivations individuelles, le phénomène social est traversé de significations dont la genèse et l'évolution sont perceptibles grâce à l'analyse historique. Expliquer signifie relier un état présent à son antécédent, reconstituer ces états afin de comprendre pourquoi, dans une situation donnée, tel type de comportement et pas tel autre a été adopté, et quelles conséquences a entraîné ce choix. Cela veut dire que la reconstitution de ces états est possible en tout temps, en cas d'accroissement ou de modification des situations qui les génèrent. Ce qui signifie qu'il n'existe pas de lois ou d'uniformités données une fois pour toutes, et que les explications avancées ne sont que des modèles, des paradigmes provisoires. Ainsi les prétentions de la sociologie au statut de science «dure» sont considérablement réduites. Il est alors

Il suffit de se référer à P. Veyne, Comment on écrit l' histoire augmenté de Foucault révolutionne l' histoire, Paris, Seuil, 1971, e Contestation de la sociologie, «Diogène», n. 75, juillet-septembre 1971, pp. 3-25. Ces travaux ont été discutés par R. Aron, Comment l'historien écrit l'épistémologie. A propos du livre de Paul Veyne, «Annales E.S.C.», 26, 1971, n. 6, pp. 1319-1354 (essai reproduit in Introduction à la philosophie de l' histoire. Essai sur les limites de l'objectivité historique. Nouvelle édition revue et annotée par S. Mesure, Paris, Gallimard, 1986) et par M. de Certeau, Une épistémologie de transition: Paul Veyne, «Annales E.S.C.», 27, 1972, n. 6, pp. 1317-1327. 
impossible de la différencier de l'histoire, mais il est aisé de la réduire à l'économie ${ }^{65}$.

Ces changements intervenus chez les sociologues ont été immédiatement enregistrés par les historiens. La mise en évidence de l'expérience vécue, des processus d'objectivation et d'intériorisation des signes, des normes et des règles, «offre la possibilité d'entreprendre une réflexion approfondie sur la dynamique des sociétés et sur l'aspect cumulatif de l'histoire humaine. Si les hommes disparaissent, les traces qu'ils ont laissées de leur passage - non seulement les traces matérielles, mais aussi les techniques, les conduites affectives et les formes d'interdépendance qui les lient entre eux - demeurent. Ceux qui leur succèdent les trouvent dès la naissance dans leur univers familier, se les approprient sans même s'en rendre compte, consacrent ainsi l'essentiel de leur vie à les développer $»^{66}$. En outre, si le chercheur peut construire, problématiser et projeter sa subjectivité sur son objet de recherche, ce n'est pas pour adopter le point de vue herméneutique de l'explication compréhensive, mais plutôt pour rejeter l'événement et son récit. Ainsi on inclut dans les procédures de contrôle empirique le point de vue subjectiviste du social, c'est-à-dire l'expérience de vérité dans l'acte de juger effectué par les acteurs et les spectateurs. L'analyse des discours, la rhétorique, la question du récit, la narration en tant que schéma explicatif va se trouver au coeur de la réflexion ${ }^{67}$.

\section{DES PROBLÉMATIQUES COMMUNES}

Si la distance entre les deux disciplines s'est réduite, aujourd'hui il est encore difficile d'en prévoir l'annulation. Chaque discipline défend son droit à l'existence, à sa tradition, à son style de travail, et cela influe sur les questions épistémologiques et sur les débats méthodologiques, sur l'orgueilleuse revendication des particularités respectives. Entre les raisons de la théorie et celles de la pratique, c'est toujours cette dernière qui a le dessus ${ }^{68}$.

65 Pour des développements substantiels il faut lire le recueil d'articles La théorie du choix rationnel. Les «Foundations of social Theory» de James S. Coleman en débat. Etudes réunies par A. Bouvier et Ph. Steiner, in «Revue française de sociologie», avril-juin 2003, pp.205-398.

66 G. Noiriel, Pour une approche subjectiviste du social, «Annales », novembre-décembre 1989, pp. 1435-1439 et du même Penser avec, penser contre. Itinéraire d' un historien, Paris, Ed. du Seuil, 2003. Sur certains développements de cette approche est à lire Jeux d'échelles. La micro-analyse de l'expérience. Textes rassemblés par J. Revel, Paris, Gallimard/Le Seuil, 1996.

${ }_{67}$ R. Robin, Histoire et linguistique, Paris, Colin, 1973; R. Chartier, J. Le Goff, J. Revel (dir.), La nouvelle histoire, Paris, Retz/CÈPL, 1978; G. Eley, De l'histoire sociale au 'tournant linguistique' dans l' historiographie américaine des années 1980, «Genèse», 7, mars 1992; P. Ricoeur, Histoire et Rhétorique, «Diogène», n. 168, octobre-décembre 1994, pp. 9-26; F. Dosse, Paul Ricoeur révolutionne l' histoire, «EspacesTemps Les Cahiers», nn. 59-60-61, 1995, pp. 6-26.

68 J. Revel, Histoire et sciences sociales: une confrontation instable, dans Passés recomposés. Champs et chantiers de l' histoire. Dirigée par J. Boutier et D. Julia, Paris, Autrement/Série Mutations n. 150-151, pp. 69-81; E. Vigne, Des emprunts raisonnés, entre disciplines, «EspacesTemps Les Cahiers », n. 59-60-61, 1995, pp. 70-77; G. Galasso, Niente altro che storia. Saggi di teoria e metodologia della storia, Bologna, Il Mulino, 2000. 
Abordons maintenant, sans aucune prétention de systématicité, quelques problématiques récurrentes dans les débats épistémologiques, et qui sont utilisées pour souligner tantôt les possibilités tantôt les impossibilités de convergence ou de divergence entre sociologie et histoire. Nous nous limiterons à en analyser deux ou trois parmi les plus significatives, ou mieux parmi celles qui sont le plus âprement discutées.

Un avertissement liminaire semble toutefois nécessaire: l'histoire et la sociologie ne disposent pas de théories générales semblables à celles de la physique, ou même à celles de l'économie, du type par exemple de l'équilibre général. Mais aussi bien l'histoire que la sociologie reconnaissent que les sociétés changent, qu'elles ont très souvent conscience de ces changements, et qu'elles cherchent parfois délibérément à les orienter et à les contrôler. Aussi bien l'histoire que la sociologie partagent une même conception de l'historicité, à savoir que l'homme est un être social marqué du sceau d'appartenance à une société dont les processus de socialisation sont produits et reproduits en un moment et en un lieu donnés. Cet être social pense le passé, il s'en sert pour forger son identité personnelle et sociale, il s'en distancie parfois, et sur la base de l'acceptation de ce passé « dépassé » il parvient même à se construire un avenir. L'action sociale est toujours action d'un individu socialisé intégré à une situation qui s'est constituée indépendamment de sa volonté, un individu socialisé ayant des objectifs élaborés dans un contexte déterminé.

Ceci étant, la connaissance de la réalité sociale est donc une connaissance d'objets spécifiques et significatifs se référant toujours à des expériences vécues. C'est de ce trait significatif de l'objet que proviennent les caractéristiques spécifiques de la connaissance historico-sociale. Quelles sont-elles?

Les comportements peuvent être sériels, c'est-à-dire collectifs, mais les décisions individuelles ont toujours une importance considérable. Les objets historiques et sociologiques sont des unités construites, des ensembles qui prennent place dans une suite. Il n'est pas toujours possible d'attribuer l'effet d'un phénomène à une cause. C'est pourquoi l'explication, dans les deux disciplines, est parfois couplée à la compréhension, et parfois même elle la remplace. Le général, le collectif est la résultante d'un effet de composition d'événements uniques et singuliers, ce qui fait que les relations individuel / collectif, micro / macro se déterminent réciproquement. De plus, l'explication et la compréhension découlent de la perspective adoptée par le chercheur, lui aussi être social enraciné dans une société donnée et regardant le passé du point de vue de l'idéologie de sa société d'appartenance. Arrêtons-nous brièvement sur l'objectivité et parlons-en non d'un point de vue philosophique, mais de celui, plus prosaïque, du chercheur œuvrant dans un domaine précis.

De quelle manière l'historien et le sociologue conçoivent-ils, dans leurs activités quotidiennes, l'objectivité? Selon quelles modalités la pratiquent-ils et la contrôlent-ils?

Pour la grande majorité des chercheurs le modèle par excellence est celui qui a été élaboré par la physique. Le néo-positivisme, dans ses différentes versions, reste la philosophie implicite de presque tous les chercheurs des sciences humaines. L'imprégnation fondamentale se fait à travers la méthodologie, réduite - très souvent - à diverses procédures techniques, à savoir aux techniques d'enquête ou aux documents d'archives. Certes, ces mêmes chercheurs ne méconnaissent pas le 
«Verstehen», mais ont tendance à s'en méfier. Par rapport à l'explication, la compréhension ne produirait aucune vraie connaissance. Tout au plus ne sauraitelle que confirmer le déjà su, le déjà connu. Elle ne garantirait - en aucun cas - la vérification empirique. Par conséquence, l'authentique forme d'objectivité reste celle de la physique où l'objectivité est indépendante du sujet, où elle n'appartient qu'à l'objet.

Souvent les chercheurs ne parviennent pas, avec les méthodes classiques, à décrire fidèlement, à expliquer les comportements des membres d'autres sociétés, notamment lorsque deux événements identiques du point de vue du comportement présentent une signification et des conséquences différentes et lorsque l'observateur est confronté aux intentions, aux mobiles du sujet impliqué, à son expérience intime, à l'action individuelle, à l'imprévu, au changement, aux mutations. C'est pourquoi ils attribuent à l'objet, moyennant certaines opérations ou mensurations, des propriétés intrinsèques, fixes, permanentes, intelligibles pour tous, impersonnelles, vérifiables. Ces propriétés, évidemment, n'appartiennent pas à l'objet observé. Elles sont des représentations que le sujet se donne et se fait de l'objet observé. Est-ce donc la validité de la représentation qui constitue l'objectivité?

Dans les sciences sociales les choses sont beaucoup plus complexes. Par exemple, les sondages d'opinion avec leurs techniques d'échantillonnage statistique, avec leurs questionnaires, prétendent analyser l'opinion commune des membres d'un groupe. En réalité, ils construisent des situations par rapport auxquelles il faut susciter des réactions d'approbation ou de désapprobation. On fabrique ainsi des artefacts, auxquels on attribue par la suite une existence objective.

Tous les phénomènes sociaux sont la résultante de constructions sociales. Ils existent en fonction de classifications provenant de nos bricolages intellectuels et des définitions imposées au chercheur par des systèmes conceptuels en vigueur dans la société. Avec des mots ou des ensembles de mots nous définissons la classification, et avec des mots provenant du découpage nous attestons ensuite l'existence de l'objet. Contrairement à ce qui se produit dans le modèle de la physique classique, dans les sciences sociales il y a impossibilité absolue de se rapporter aux choses pour expliquer les signes. Nous construisons avec des définitions sociales les objets étudiés, de même que leurs caractéristiques et leurs contenus. Au moment où nous les saisissons, nous constatons qu'ils sont identiques à ceux que nous avions posés au départ de la recherche.

La théorie dans les sciences humaines n'a ni la même nature ni les mêmes fonctions que celles que l'on observe, par contre, dans les sciences empiricoformelles. Dans les sciences sociales la déduction est difficilement praticable. La logique démonstrative cède régulièrement la place à la logique argumentative. Les conceptualisations qui, grâce aux règles de correspondance, devraient être traduites en propositions empiriques, ne peuvent pas l'être.

De chercheurs, à partir de la fin des années '60, ont tenté de préciser ce que pourraient être des modalités cohérentes pour interroger, pour construire ou pour reconstruire la réalité humaine moyennant des concepts et des représentations malgré les nombreuses difficultés et perplexités. Si la connaissance est une construction / reconstruction du réel au moyen de concepts élaborés par des sujets, nous réduisons l'objectivité à la validité de ces constructions conceptuelles ou de 
ces représentations. Si on admet la légitimité de cette réduction, il faut résoudre le problème de la sélection inhérente à ce type de conceptualisation. Or, dans le vaste univers des relations sociales et des systèmes culturels, il faut choisir, séparer, sélectionner, éliminer. Dès lors, la connaissance produite est fragmentaire, désarticulée, partielle, non finie. N'étant ni absolue ni totale, comment pouvons-nous encore parler d'objectivité?

A ce propos, on peut observer deux attitudes différentes par rapport à cette problématique. La première attitude présuppose que l'objectivité est un idéal, un désir infini, l'expression d'intentions d'ordre psychologique ou social de détachement, le contraire d'une implication sereine et pondérée. L'objectivité serait une sorte de désengagement, d'indépendance par rapport au vécu, aux particularités culturelles, aux contingences historiques, aux conditionnements et aux déterminations de l'environnement socioculturel. La seconde fait de l'objectivité une hypostase de l'impartialité et de l'équité. Le chercheur est un homme sans préjugés, sans appartenances partisanes, capable de pondérer les forces en jeu, de les évaluer de manière équitable et équilibrée.

Dans les deux cas il y a objectivité lorsque le scientifique parvient à se détourner de la subjectivité, c'est-à-dire de ses préférences, de ses intérêts, des rapports de force qui l'enserrent plus ou moins. De quelle façon obtenir le détachement, garantir la non implication alors même qu'on est confronté à des significations, à des croyances, à des opinions, à des normes, à des vérités constitutives de notre identité sociale? Jean Piaget parle de décentration, d'une inversion des rapports sujet-objet et de la construction d'un système de réciprocité qualitativement nouveau par rapport à l'action initiale. Ceci revient à dire que le passage de la subjectivité à l'objectivité exige une dissociation perceptive, intuitive, affective et intellectuelle par rapport à l'action, qu'il faut ensuite resituer dans un contexte de relations spatiales et causales indépendantes de l'activité immédiate, et selon des procédures intersubjectives.

Cette décentration (générale ou d'ensemble) offre-t-elle des garanties réelles contre les illusions des sens, du langage, des points de vue, des préjugés ethniques et des préventions culturelles? Comment débusquer et faire disparaître toutes les retenues, les empêchements, les obstacles du moi, distancier des sagesses produites par les conditions et les positions de classe? Comment être certains de ne pas confondre la perspective personnelle avec la perspective absolue et immédiatement objective? La décentration, le détachement, la sérénité, l'impartialité, difficilement réalisables dans les petites comme dans les grandes circonstances, relèvent du devoir du chercheur mais ne sont en aucun cas des caractères ou des caractéristiques de la science. Dire que la science est objective parce que le chercheur est impartial ou décentré revient à affirmer que l'objectivité se confond avec l'élimination de toute subjectivité, mais en omettant de dire quels seraient les moyens permettant au scientifique d'acquérir un regard libéré des impuretés et des faiblesses de la subjectivité.

Depuis que nous savons que la permanence des formes ou des catégories, peut-être même de certaines règles logiques, n'est point l'expression d'une raison absolue; depuis que nous savons que les formes de la sensibilité ne sont pas universellement humaines, que les catégories ne sont pas fixées une fois pour toutes parce que l'intellect est fini et limité; bref, depuis que nous connaissons un peu mieux les mécanismes à travers lesquels le sujet construit l'objet, ainsi que les 
relations interactives qui s'établissent entre le sujet et l'objet, nous sommes devenus plus exigeants quant aux modalités d'élaboration des savoirs. Des chercheurs conscients des liens existant entre l'objet construit et le sujet qui interroge le réel et en tire des corrélations, conçoivent l'objectivité comme une qualité particulière d'une connaissance convenablement ordonnée par rapport aux caractéristiques de l'objet construit. Généralement, cet ordre convenable correspond à l'adéquation consciente du soi et de l'autocritique. Mais cette adéquation reste très problématique car la dépendance des objets par rapport aux actes et aux opérations successives les saisissant, les fixant et les définissant, reste un mystère. S'il était possible de saisir, dans l'acte de connaissance, un contenu indépendant de l'acte mental à travers lequel on s'efforce de comprendre-expliquer les propriétés des objets; si les moyens utilisés ne transformaient pas les objets à connaître, alors les réticences à l'égard de l'objectivité en tant que qualité d'un savoir, d'une connaissance convenablement ordonnée, seraient non pertinentes.

La critique de l'objectivisme et la constatation que souvent la distanciation et l'impartialité investissent l'objet observé de qualités passives, statiques, abstraites, séparant artificiellement l'observateur de l'observé, - voilà qui incite de plus en plus certains chercheurs à remplacer la notion d'objectivité par celle d'objectivation. Toutes les sciences sociales disposent de processus d'objectivation produisant non pas un savoir vrai, général, universel, mais plutôt un savoir valide, c'est-à-dire cohérent, qui n'est affecté d'aucune clause de nullité. Ce savoir est valide indépendamment de la vérité de ses propositions, dans la mesure où il n'est pas en contradiction avec le niveau moyen des savoirs et des connaissances en vigueur à ce moment-là dans la discipline, avec les critères, légitimes et valides, adoptés par la communauté scientifique. La procédure d'objectivation est une construction dépendante d'une interprétation préliminaire qui conditionne la signification des résultats. Cette procédure n'est pas une simple modification dans la façon de comprendre les objets, c'est une pratique qui remplace un univers par un autre univers. Au vécu elle substitue le construit; à l'opacité et à l'ambiguiité du perçu, la transparence des modèles. L'erreur, l'hypothèse, le doute, l'hésitation deviennent ainsi des éléments constitutifs et essentiels d'un processus interminable, inépuisable.

Le point délicat de cette conception de l'objectivité est que le contenu, dans cette façon de procéder, dépend des critères d'acceptabilité, et ceux-ci reposent sur un ensemble de valeurs cognitives fixant la légitimité de certaines représentations.

Ces conceptions, compréhensibles à la lumière des expériences de recherche de terrain, mettent néanmoins en évidence que le dualisme entre l'objectivité universellement admise et la subjectivité incommunicable, entre la réalité qui s'impose à tous et les valeurs individuelles et arbitraires, entre les vérités nécessaires et éternelles et les savoirs soumis aux contingences sociales et historiques, entre une rationalité technoscientifique et un décisionnisme existentiel réduisant les choix à des décisions irrationnelles et à des actes de foi, - que ce dualisme est trop rigide. Le domaine des sciences sociales comprend ce que les différentes générations d'hommes ont vécu et créé, ce qu'ils ont fait et ce qu'ils font, comment ils le font dans le temps, c'est-à-dire dans l'imprévu, dans le risque, dans l'anticipation. L'objectif ultime de ces sciences est également bien déterminé: décrire, comprendre, interpréter la réalité humaine, le sens subjectif des conduites 
sociales au moyen de récits, de narrations rendant intelligible ce qui n'est pas apparent, manifeste, ou empiriquement saisissable. Cela signifie que dans toutes les sciences sociales - peut-être pour des raisons tenant à l'ontologie de la réalité historico-sociale - la signification joue un rôle primordial. Parler de phénomènes humains revient, par conséquence, à parler de sens et de significations. L'identité entre le sujet et l'objet, justement pour cette raison, demeure cruciale. Le sujetchercheur ne déchiffre pas une intelligibilité immanente, mais la construit en choisissant entre de multiples systèmes de référence et des points de vue disparates. Si toute reconstruction est un choix, la pluralité d'interprétations applicables à un même ensemble est inévitable. Ces reconstructions partielles d'une réalité globale dérivent d'un système de concepts que nous pouvons justifier mais pas vérifier. Pour cela les représentations élaborées sont mouvantes, variables, susceptibles d'adjonctions, de compléments, de changements. Comprendre objectivement le sens subjectif des conduites sociales signifie édifier hypothétiquement un sens parmi plusieurs également possibles. La connaissance ainsi acquise modifie tout à la fois les caractéristiques de l'objet et le sujet qui interprète. Ces interprétations multiples d'un ensemble permettent de faire ressortir les questions qu'elles expriment, mais pas de les additionner ni de les cumuler. Et pourtant ces mêmes questions sont régulièrement reprises dans les interprétations suivantes et sont ainsi intégrées dans les nouvelles constructions. La diversité des questions, la diversité des méthodes, les compréhensions successives et multiples, la pluralité des plans de référence privent les sciences sociales de théories déductives mais non de la possibilité de justifier rationnellement, moyennant des normes reconnues intersubjectivement, leurs constructions théoriques.

Toutes les sciences disposent de modèles discursifs de validation des normes. Ces modèles permettent d'élaborer des théories, de les critiquer, de les justifier et parfois même (c'est le cas en économie) d'en démontrer le caractère probabiliste. Le langage, les méthodes, les problèmes, les théories des sciences sociales constituent un corpus autour duquel s'est formé un consensus, un paradigme au sens de Th. Kuhn. Les théories des sciences sociales sont des instruments puissants pour argumenter la subjectivité de façon plausible, raisonnable et parfois logique. Elles sont des schématisations permettant de rationaliser certains contenus et certaines représentations. Diverses opérations de construction et de cohésion rendent les schématisations acceptables et recevables. Bien entendu, la validité d'une représentation est toujours susceptible d'être corrigée car la cohérence et la justesse d'une construction ne représentent jamais un état absolu et définitif.

Les sciences sociales sont en mesure de ne pas se laisser submerger par une subjectivité incontrôlée; elles peuvent établir des relations et des corrélations solides en assurant ou en garantissant la validité de tout ce qui dans les conduites humaines peut être représenté de manière plausible et raisonnable, totalement ou partiellement.

Au-delà des tensions entre le sujet connaissant et l'objet du connaître; au-delà du pluralisme des interprétations, des situations empreintes de subjectivisme et de relativisme; au-delà du fait que toutes les sciences sociales sont régies principalement par la logique de l'argumentation et par la rhétorique, il faut reconnaître la validité de leurs représentations. Soit que l'histoire et la sociologie affrontent les mêmes questions, celles qui se rapportent à la validité des représentations, et que les deux sont confrontées aux mêmes difficultés, à ces mêmes mystères que les comportements sociaux n'arrêtent jamais de générer et d'embrouiller. 
Le problème du temps suscite d'âpres controverses entre les sociologues et les historiens. Les uns et les autres tiennent pour essentielle la conception du temps comme ordre fondamental du mouvement mais ils n'ont pas la même manière de le traiter. Les sociologues croient que les historiens conçoivent le temps comme une suite de phénomènes, d'événements reliés les uns aux autres, et présument que l'explication historique consiste en un récit de cette suite et de ces connexions. Les historiens considèrent que les sociologues expliquent les phénomènes sociaux comme s'ils étaient dépourvus de genèse et d'évolution, indépendants de l'ordre de succession, comme s'ils étaient des totalités produites uniquement par des systèmes de relations présentes.

La dispute n'a pas de fondements réels. Les sociologues ont toujours su distinguer le mode de constitution et d'évolution d'un phénomène de ses fonctions à un moment déterminé. Il suffit de se rappeler les recherches de Claude Lévi-Strauss sur Les structures élémentaires de la parenté, ou sur les mythes, ou encore celles de Talcott Parsons sur la crise de l'université. En ce qui concerne les historiens, personne ne peut dire que La civilisation de la Renaissance en Italie de Burckhardt ou La société féodale de Marc Bloch soient de simples narrations d'une suite d'événements. Leurs reconstructions des types de société visent, sans aucun doute, à retrouver le sens, la structure, l'organisation, le système de valeurs, les principes informateurs, la singularité de la culture de la Renaissance italienne et de la Société médiévale.

La dispute et ces désaccords proviennent d'un choix analytique. Les historiens privilégient la diachronie tandis que les sociologues la synchronie. Les premiers attachent une grande importance à la succession et à la consécution; les seconds à la simultanéité des relations à un moment déterminé. Ces modalités ne sont pas opposées, mais seulement distinctes. Quand l'historien, par exemple, écrit l'histoire de la Révolution industrielle, il subsume sous la même catégorie des événements de la période 1750-1820. La première période est caractérisée par l'usage massif des métiers à tisser mécaniques, la deuxième par l'utilisation de la vapeur, la troisième de l'électricité. La caractérisation de la première période, tout comme celle des deux autres, est obtenue à travers des analyses synchroniques. La Révolution industrielle est reconstruite grâce à la succession de trois synchronies. La reconstruction aurait été irréalisable d'un point de vue purement diachronique, étant donné que l'utilisation de l'électricité, caractérisant la période 1880-1900, ne peut être déduite ni des métiers à tisser mécaniques ni de la vapeur. Pour l'expliquer il faut définir préalablement des unités et des identités logiques. Pour rendre compte des structures temporelles, il faut recourir à ces catégories logiques, mais celles-ci restent toujours des systèmes d'équivalence entre des choses appartenant à des ordres différents ou semblables. Si sur un plan logique il est impossible d'opposer diachronie et synchronie, sur le plan pratique la distinction est utile, puisque l'explication historique et l'explication non génétique mettent en évidence des ordres différents. La genèse et les filiations aident à saisir les continuités et les permanences, mais pas à comprendre les fonctions actuelles des institutions, pour le dire avec Piaget: «... la sociogenèse des structures n'explique pas leur fonction ultérieure, car en s'intégrant dans de nouvelles totalités ces structures peuvent changer de signification. En d'autres termes, si la structure d'un concept dépend de son histoire précédente, sa valeur dépend de sa position fonctionnelle dans la totalité à laquelle il appartient à un moment donné, et ce 
n'est que lorsque l'histoire consiste en une succession de totalités orientées vers un équilibre croissant que la genèse détermine la valeur actuelle des notions ${ }^{69}$.

Piaget pense qu'il serait contreproductif d'absorber le synchronique dans le diachronique (comme le souhaiteraient les historiens) ou le diachronique dans le synchronique (comme le suggèrent certains sociologues). La première assimilation n'augmenterait certainement pas la puissance explicative du modèle causal; la seconde ne modifierait pas la nature de l'implication, c'est-à-dire la connexion de l'antécédent au conséquent. Pour dépasser la dualité entre facteurs diachroniques et synchroniques, il faudrait découvrir les voies permettant de passer de la causalité à l'implication. L'analyse synchronique, qui privilégie les relations de simultanéité ou de dépendance réciproque, fait abstraction de la temporalité, met en évidence les structures logiques d'un ensemble, d'un système. Cette analyse n'est pas inconnue des historiens (Burckhardt, Bloch) mais elle est peu pratiquée, parce que le mode d'exposition courant en historiographie reste la narration comme succession d'événements. En d'autres termes, le changement, comme passage d'un stade à l'autre, d'une forme à l'autre, reste le noyau dur de l'historiographie. A l'opposé, les fonctions semblent être moins influencées par les changements, parce qu'elles ne concernent pas des expériences ou des réalités vécues, mais des interdépendances de rôles d'organisations, c'est-à-dire de systèmes, d'ensembles, de collectifs idéaux. L'histoire de la famille, de la Restauration à nos jours, s'écrit autrement que la sociologie des fonctions familiales dans les sociétés post-industrielles. Mais les fonctions d'aujourd'hui sont la cristallisation de toutes les mutations ayant affecté les structures au cours des siècles. Pourquoi ces différences de forme sont devenues avec le temps déterminantes; pourquoi nous sommes impuissants à effacer les distances, accentuées par les situations académiques respectives et par les luttes pour la survie dans les institutions universitaires?

L'histoire des théories sociologiques, des représentations de la réalité, de leurs évolutions, montre qu'une certaine conception de la société et de ses mouvements a favorisé la diffusion et puis assuré l'hégémonie des analyses synchroniques. Elle a fait proliférer une théorie créant des faits et puis, grâce à un mouvement circulaire, exigeant d'eux l'épreuve des faits, la vérification par l'observation et le raisonnement, en bref sa propre vérification.

On sait que les faits ne sont pas inscrits dans le monde qui nous entoure ni dans notre monde intérieur; il est donc futile d'imaginer que l'on puisse enregistrer l'existence de faits, lesquels résultent toujours d'un ensemble d'opérations mentales d'organisation, de mise en relation, de complexification. Par ailleurs, même si l'on admettait un instant qu'il y ait quelque part des faits donnés une fois pour toutes, ceux-ci ne suffiraient pas à supporter les déductions. La logique du

J. Piaget, Problèmes généraux de la recherche interdisciplinaire et mécanismes communs, in Tendances principales de la recherche dans les sciences sociales et humaines. Première partie: Sciences sociales, préface de R. Maheu, Paris, Unesco, 1970, pp. 588-589. Lire également du même la préface au volume Epistémologie des sciences de l'homme, Paris, Gallimard, 1970, à l'origine de multiples discussions: G. Busino hrg., Jean Piaget-Werk und Wirkung, München, Kindler, 1976, et Struktur und Vernunft. Jean Piaget Modell entwickelten Denkens in der Diskussion Kulturvergleichender Forschung. Hrg. Von T. Schöfthaler und D. Goldschmidt, Frankfzurt a.M., Suhrkamp, 1984. 
quotidien et l'importance des discours pratiques sont évidents. Pour connaître les mécanismes susceptibles de les produire, les recherches sur le discours argumentatif tentent de déchiffrer les modalités du fonctionnement de la logique naturelle, de faire comprendre pourquoi les sciences de l'homme et de la société font constamment appel aux analogies, aux métaphores et aux métonymies. La raison pratique, celle qui vit dans l'histoire, est gouvernée par des lois informulables immédiatement en termes mathématiques. Dans les sciences «pratiques et poétiques » aristotéliciennes sont à l'œuvre d'autres rationalités. Il est facile, pour n'importe quel problème, d'énoncer des structures puis de les doter de formes logico-mathématiques, mais cela ne peut se faire qu'à l'intérieur du paradigme booléen. L'écart entre l'algèbre de Boole et la pensée naturelle, la donnée que pour saisir la forme des faits et des processus il faille toujours recourir au non formel, ou encore que pour expliquer les systèmes formels dépourvus et de sujets et de critères d'interprétation on doive recourir à des aides extérieures, - ce sont des hypothèses communes à l'histoire et aux sciences sociales qui commencent à être admises par les sociologues et par les historiens. La redécouverte du sens des pratiques sociales rapproche l'histoire et les sciences sociales. Et il n'est pas improbable que de cette collaboration puisse naître une authentique science de l'homme et de la société.

\section{LES APPORTS DE L'HISTOIRE}

Il n'y a pas de domaine en sociologie où l'on puisse faire l'économie de l'histoire. Même à notre époque où l'histoire avance par bonds et où la discontinuité semble détruire ce que Jean Piaget appelle la pression d'une génération sur l'autre, le rôle de l'histoire reste, selon Wright Mills ${ }^{70}$ essentiel pour la sociologie. L'histoire est la mémoire organisée de l'humanité, l'immense dossier indispensable à toute science sociale car aucune société n'est compréhensible sans l'apport des matériaux historiques tels, par exemple, que ceux réunis dans les livres de $\mathrm{Ph}$. Ariès ${ }^{71}$, de P. Laslett ${ }^{72}$ ou d'O. Dumoulin ${ }^{73}$ qui sont des exemples significatifs pour connaître les traditions, les attaches et tous les autres facteurs qui ont contribué, tout au long de la vie des groupes culturels, à déterminer des modèles de comportements, repris et standardisés par les institutions; pour la bonne compréhension de la famille, du fossé des générations, des cultures des jeunes, des processus de socialisation. Jean-Paul Tréanton ${ }^{74}$ a bien noté que: «Toute histoire des idées et

C. Wright Mills, The Sociological Imagination, New York, Oxford University Press, 1962, ch. VIII.

${ }^{1} \mathrm{Ph}$. Ariès, L'enfant et la vie familiale sous l'Ancien Régime, Paris, Plon, 1960; mais également Histoire des populations françaises et de leurs attitudes devant la vie depuis le XVIII siècle, Paris, Seuil, 1971; L' homme devant la mort, Paris, Seuil, 1977; Un historien du dimanche, avec la collaboration de M. Winock, Paris, Seuil, 1980; Le temps de l' histoire. Préface de R. Chartier, Paris, Seuil, 1986; Essais de mémoire, 1943-1983. Avant-propos de R. Chartier. Paris, Seuil, 1993; Le présent quotidien, 1955-1966. Introduction et notes de J. Verdès-Leroux, Paris, Seuil, 1997.

72 P. Laslett, Un monde que nous avons perdu. Famille, communauté et structure sociale dans l'Angleterre pré-industrielle, Paris, Flammarion, 1969.

73 O. Dumoulin, Le rôle social de l'historien. De la chaire au prétoire, Paris, Albin Michel, 2003.

74 J.R. Treanton, Le sociologue est-il en droit d'ignorer l'histoire?, «Revue française de sociologie », janvier-mars 1970, pp. 94-99. 
des pratiques sociales (c'est bien de cela qu'il est question sous couvert de sociologie) apparaît comme une entreprise vouée à l'échec si son auteur ne la situe pas, avec scrupule dans le contexte matériel et moral de l'époque.» En effet, chaque société se saisit et s'analyse elle-même à travers son histoire. Ceci induit un certain type de connaissance moyennant laquelle nous rendons compte des raisons, des mobiles, des intentions, des objectifs et des stratégies des acteurs, de ce qu'ils disent de leur vécu, de leurs expériences, des mécanismes collectifs socialement institués et organisés, qui font fonctionner les systèmes procéduraux de perception, d'appréciation, de médiations, d'attribution du sens et des orientations.

L'histoire peut éviter à la sociologie de fixer l'attention exclusivement sur les types idéaux, sur les imputations causales, sur les structures et les systèmes statiques vus à court terme. Elle peut l'aider à mieux évaluer les événements et les comportements individuels, à élaborer des questionnements à partir d'héritages réels, de références situées, de particularités locales, à distinguer les activités intentionnelles des résultats atteints, les phénomènes volontaires de ceux inattendus, les agrégations spontanées de celles volontaires et exploratoires, en bref, de ne pas transformer le descriptif en explicatif. Etant donné que les faits sociaux revêtent une signification variable selon la position sociale et historique de l'acteur et de l'observateur et que l'un et l'autre ont conscience ou connaissance du caractère foncièrement historique de leur être au monde, il est important pour les sociologues d'utiliser avec précaution les concepts de changements, d'évolution, évolutionnisme, mutation et révolution ${ }^{75}$. En plus, l'histoire indique aux sociologues la dimension historico-sociale des procédures par lesquelles on emporte la conviction d'un auditoire mais aussi des procédures par lesquelles on s'autopersuade. Il en est de même pour l'expérience de la durée, pour le temps tel qu'il est vécu, pour le temps qui n'est inscrit dans aucun ordre de nécessité ou dans un passé donnant du sens aux projets individuels et collectifs ${ }^{76}$.

A un moment où les technologies bouleversent les façons de faire de la recherche, à un moment où les banques de données et les traitements électroniques des données instaurent des pratiques contraignantes, la méthode historique, l'analyse méthodique des témoignages, l'établissement critique des faits et leur évaluation, l'érudition historique nous aident à écarter des banques de données les futilités, à situer et à évaluer les «trouvailles» prétendument nouvelles ${ }^{77}$. Les travaux de Lazarsfeld sur histoire de la quantification ${ }^{78}$, ceux d'Oberschall sur la

75 R. Boudon, La place du désordre. Critique des théories du changement social, Paris, Quadrige/ Puf, 1991.

76 E. Zerubavel, Hidden Rhythms. Schedules and Caldendars in Social Life, Chicago, University Press, 1981; H. Nowotny, Eigenzeit: Entstehung und Strukturierung eines Zeitgefühls, Francfurt a.M., Suhrkamp, 1989; D. S. Milo, Trahir le Temps, Paris, Les Belles Lettres, 1991.

77 Pour le parti qu'on peut tirer de la méthode historique, v. l'article de J. Bourdon, La critique historique appliquée aux documents statistiques et numériques, «Journal de la Société de statistique de Paris », 97, n. 1-2-3, janvier-février mars 1956, pp. 24-49.

78 Cf. P.F. Lazarsfeld, La philosophie des sciences sociales, Paris, Gallimard, 1970, et les études réunies soua la direction de J. Lautman et B.-P. Lécuyer, Paul Lazarsfeld (1901-1976). La sociologie de Vienne à New York, Paris, L'Harmattan, 1998. 
recherche empirique ${ }^{79}$ ont révélé, grâce à l'histoire, l'origine et la véritable portée de l'analyse empirique de l'action sociale. Par ailleurs, une certaine pratique de la méthodologie a accrédité le mythe de l'efficacité absolue des techniques et de la neutralité des méthodes logico-mathématiques. L'histoire permet d'entrevoir les intérêts extrascientifiques et les conditionnements sociaux que telle ou telle autre méthode véhicule ou a véhiculés, comment une méthode a été interprétée, accueillie, travestie par les forces sociales, et quel usage en ont fait les sociologues. Grâce aux études historiques, il est possible d'évaluer à la fois la portée sociale d'une méthode et de déceler les conditions nécessaires pour qu'elle produise des résultats dans certaines situations. L'histoire des typologies dit comment et pourquoi on a construit les types idéaux, c'est-à-dire des modèles conceptuels stylisant les traits essentiels d'une individualité historique, d'une institution, d'une relation sociale, pour en dégager l'intelligibilité ${ }^{80}$. On pourrait également ajouter que les livres d'histoire peuvent donner une profondeur spatiotemporelle aux théories macrosociologiques. L'extension des sondages sociologiques a entraîné certaines dysfonctions ou simplement certaines insuffisances dans la recherche sociologique, entraînant certaines déficiences dans les techniques de l'analyse secondaire, l'histoire peut aider à bien utiliser les données de nature et de composition très diverses ${ }^{81}$.

L'apport le plus important de l'histoire à la sociologie se situe au niveau de l'analyse comparée ${ }^{82}$. Celle-ci, grâce aux jeux de rapprochements entre des situations concrètes, aux analyses des identités et des différences, grâce à l'étude des variations concomitantes repérant les similitudes, à la confrontation de la réalité observée avec le modèle théorique, vise à découvrir les éléments constants, à dégager des types, à fonder l'expérimentation indirecte. Avec la méthode comparative on étudie les processus sociaux et politiques, les institutions, les organisations, de façon synchronique ou diachronique, à temps égaux, ou donnés en tant que tels, ou encore à temps différents mais réputés appartenir à des ordres sociaux quasi semblables. La comparaison synchronique permet l'utilisation de la condition cateris paribus alors que celle diachronique, longitudinale, se fonde inévitablement sur la logique culturelle du chercheur, sur un certain système de valeurs, sur l'utilisation de catégories analytiques non pertinentes. Ce type de comparaison, déjà largement utilisée par les tenants d'un développement d'inspiration fonctionnaliste ${ }^{83}$, par des durkheimiens

79 A. R. Oberschall, The Establishment of Empirical Sociology. Studies in continuity, discontinuity and institutionalization, New York, Harper \& Row, 1972, et Empirical Social Research in Germany, 1848-1914, Paris, Mouton, 1965.

so D. Schnapper, La compréhension sociologique. Démarche de l'analyse typologique, Paris, PUF, 1999.

81 R. Boudon, La crise de la sociologie. Questions d'épistémologie sociologique, Genève, Droz, 1971.

82 Cf. la bibliographie de R.M. Marsh, Comparative Sociology, New York, Harcourt \& Brace, 1967, et le 'reader': Comparative Methods in Sociology. Essays on Trends and Applications, edited by I. Vallier, Berkeley, University of California Press, 1971.

83 S.N. Eisenstadt, Comparative social problems, New York, Free Press, 1964; Essays on comparative institutions, New York, J. Wiley, 1965; Modernization: protest and change, Englewood, Prentice-Hall, 1966; Comparative perspectives on social change, Boston, Little, Brown, 1968; The Protestant ethic and modernization; a comparative view, New York, Basic Books, 1968. 
ou wébériens (Lipset ${ }^{84}$, Rokkan ${ }^{85}$ et Almond ${ }^{86}$, Tilly ${ }^{87}$, Bendix ${ }^{88}$ ), par des néomarxistes ${ }^{89}$, établit des relations entre des éléments structuraux de systèmes globaux différents. Des exemples excellents ont été fournis par l'étude de Charles E. Frye ${ }^{90}$ à propos des relations empiriques entre le système des partis, les groupes de pressions et la stabilité politique en Allemagne à l'époque de la République de Weimar et puis de la R.F.A., par les travaux de Gino Germani ${ }^{91}$ sur la modernisation des sociétés, par Raymond Aron sur la guerre et la paix ${ }^{92}$ ou de Jean Baechler sur les phénomènes révolutionnaires, sur le capitalisme, sur les suicides, sur l'idéologie, sur les démocraties, sur le régime des castes ou sur les traits distinctifs de l'espèce humaine ${ }^{93}$.

Toutes ces recherches postulent que les variables explicatives sont indépendantes des cultures propres aux objets à analyser, qu'il y a une idée universelle de l'Etat et que l'articulation du politique à la société est partout et en tous les temps la même.

L'histoire permet d'écarter ces reconstructions opérées à priori, d'obtenir en retour des éclairages réciproques nettement plus intéressants, bien qu'il faille recourir à la clause cateris non-paribus, difficile à manier car elle fonde sa prétention explicative non sur des conditions causales, sur des régularités nécessaires, sur la quête de lois, mais au contraire sur la pluralité, sur la distinction, sur le postulat que les différences et les altérités ne sont pas réductibles aux mêmes variables explicatives. Les travaux de Karl Polanyi expliquent la société moderne en la contrastant avec la société traditionnelle, ceux de Louis Dumont expliquent l'individualisme et l'égalité de la société occidentale en l'opposant à la société

84 Il suffit de citer le livre Student Politics, New York, Basic Books, 1967.

85 Cf. Party Systems and Voter Alignments, New York, Free Press, 1967, et Comparing Nations: The Use of Quantitative Data in Cross-National Research, New Haven, Yale University Press, 1966. Du même cf. également: Citizens, Elections, Parties: Approaches to the Comparative Study of the Process of Development, Oslo, Universitetsforlaget, 1970.

s6 Pour la bibliographie de G.A. Almond, cf. le livre de G. Urbani, L'analista del sistema politico, Bologna, Il Mulino, 1971.

87 Ch. Tilly, Coercion. Capital and European States AD 990-1990, Oxford, Blackwell, 1990.

88 R. Bendix, Kings or People, Berkeley, University of California Press, 1978. Voir également G. Busino, La contribution de Reinhard Bendix à l'élaboration de la sociologie historique, « Revue internationale de politique comparée », vol. 5, n. 3, 1998, pp. 507-520.

89 Il s'agit de E. Hobsbawm, Barrington Moore, Perry Anderson, Michael Hechter, Theda Skocpol, I. Wallerstein. Pour une présentation de ce courant, cf. B. Badie, Le développement politique, Paris, Economica, 1988.

9o Ch.E. Frye, Parties and Pressure Groups in Weimar and Bonn, «World Politics », XVII, 1965, pp. 635-655, mais également R. Dahrendorf, The New Germanies : Restoration, Revolution, Reconstruction, «Encounter», XXII, 1964, pp. 50-58, repris ensuite in Gesellschaft and Demokratie in Deutschland, München, Piper, 1965.

91 G. Germani, Sociologia della modernizzazione. L'esperienza dell'America Latina, Bari, Laterza, 1971, et Politique, société et modernisation, Gembloux, Duculot, 1972.

92 R. Aron, Paix et Guerre entre les Nations. Huitième édition avec une présentation inédite de l'auteur, Paris, Calman-Lévy, 1984, et Penser la guerre, Clausewitz., Paris, Gallimard, 1976, 2 vols.

93 Citons, pour mémoire, les dernières publications: J. Baechler, Nature et Histoire, Paris, PUF, 2000, et Esquisse d' une histoire universelle, Paris, Fayard, 2002. 
holiste et hiérarchique indienne. Dans les deux cas, l'utilisation de l'histoire a ouvert des perspectives nouvelles aux sciences sociales.

\section{LES APPORTS DES SCIENCES SOCIALES}

En retour, les historiens peuvent emprunter différents schémas explicatifs aux économistes et aux sociologues, notamment dans le domaine de la théorie, des techniques et des sources pour l'histoire de demain ${ }^{94}$.

L'historien est méfiant à l'endroit des théories, des concepts abstraits, plus ou moins organisés, appliqués à un domaine particulier de la connaissance. Un historien à théories est réputé dépourvu de bon sens, de réalisme, à la limite bourré de partis pris.

Or, la répulsion insurmontable, irrésistible affichée à l'endroit de la théorie est injustifiée. A la base de tout travail historique, il y a toujours une théorie, une construction méthodique et organisée, assez souvent implicite, de nature hypothético-déductive. Tout travail historique est guidé par une théorie, soit-elle implicite. Karl Popper ${ }^{95}$ nous a expliqué qu'une théorie est un filet que nous tendons pour capturer le monde, pour le rationaliser, pour l'interpréter, pour le dominer. Lorsque l'historien étudie le mariage à Genève ou en Pologne au XVII ${ }^{\mathrm{e}}$ siècle, la distribution de la propriété foncière en Bavière ou en Catalogne au XVIII ${ }^{\mathrm{e}}$ siècle, il utilise, sans le savoir peut-être, des systèmes théoriques sur la famille et sur le mariage, sur la propriété, qui lui permettent de récolter ces données, de les agencer, de les comprendre et dans certains cas de les expliquer au moyen d'un récit.

Savoir manier sciemment les théories, le cas échéant pouvoir les construire, savoir qu'une théorie est un ensemble de propositions liées les unes aux autres de manière systématique et permettant d'élaborer des généralisations - la famille, le mariage, la classe sociale, etc. - des généralisations susceptibles d'être vérifiées empiriquement, ce n'est pas un jeu intellectuel mais plutôt une nécessité pratique ${ }^{96}$.

Prenons, à titre d'exemple, un livre d'histoire sur la Révolution française, un autre sur la Révolution russe et un autre encore sur les événements de mai $1968^{97}$. Tous ces livres examinent un ensemble d'événements ayant lieu dans certaines sociétés et caractérisés par des changements importants, par des ruptures profondes. Ils se fondent sur une théorie de la révolution, laquelle permet de récolter les faits, de les classer, de les connecter, de les interpréter, de leur donner une signification ou des significations, celles de Jean Jaurès ou de Georges

${ }^{94}$ Cf. S.M. Lipset and R. Hofstadter, Sociology and History: Methods, New York, Basic Books, 1968.

95 Cf. K. Popper, The Logic of Scientific Discovery, New York, Harper Torchbooks, 1968.

96 Cf. R. Boudon, A quoi sert la notion de «structure»? Essai sur la signification de la notion de structure dans les sciences humaines, Paris, Gallimard, 1968 et Les méthodes en sociologie, Paris, PUF, 1969.

${ }_{97}$ M. Zancarelli-Fournel, 1968: histoire, mémoires et commémoration, «EspacesTemps Les Cahiers », nn. 59-60-61, 1995, pp. 146-156. 
Lefebvre $^{98}$, d'Isaac Deutscher ${ }^{99}$ ou d'Adrien Dansette ${ }^{100}$, ou encore d'Alain Touraine ${ }^{101}$. Les origines de la France contemporaine d'Hippolyte Taine, par exemple, n'ont rien en commun avec l'Histoire socialiste de la Révolution française de Jean Jaurès, car ces deux histoires fondent leurs analyses détaillées, l'évaluation critique des expressions, leurs comparaisons, l'identification dans un événement du fondamental et du contingent, leurs procédures de généralisation, sur des constructions théoriques fondamentalement disparates.

L'assassinat de César est, certes, un événement unique. Cependant, il rentre aussi dans la classe des assassinats politiques, de phénomènes qui, à côté d'éléments contingents et spécifiques, présentent des caractéristiques communes comparables.

La maîtrise des règles régissant les arrangements, les permutations et les combinaisons des éléments à l'origine des notions servant à décrire les phénomènes, offre des avantages certains pour la mise en perspective des comparaisons, pour déceler des éventuelles uniformités. Bien que celles-ci soient impropres à la prédiction, elles aident néanmoins à circonscrire les réactions qu'on peut avoir devant un événement aléatoire. Dès lors il est possible de construire des modèles non déterministes, stochastiques, des modèles qui reconnaissent la place des aspects aléatoires des phénomènes, à leurs côtés uniques et atypiques - aux réactions des hommes vis-à-vis des événements.

Par ailleurs, l'historien utilise couramment les notions de protestantisme, capitalisme, libéralisme, socialisme, économie urbaine, réalisme, néo-réalisme..., sans jamais s'interroger sur les règles de la logique ou de la logistique les ayant produites. En histoire, l'utilisation des typologies est variable et chatoyante. Tantôt le type capitalisme est un outil pour faire des comparaisons entre des époques économiques différentes, tantôt il est le «collant» de données empiriques peu structurées. En un mot, il n'y a pas de recherche sans théorie, il est dangereux d'utiliser une théorie sans la maîtrise des caractéristiques du type construit, sans l'analyse préalable de sa structure logique, sans la connaissance des relations entre les composants.

Un exemple aidera à mieux comprendre cette problématique. Pour étudier le socialisme suisse du $\mathrm{XIX}^{\mathrm{e}}$ siècle, il faut recourir à une masse énorme de documents, de données plus ou moins sûres, plus ou moins significatives, d'origine très différente, à des rapports de police, aux journaux, livres, tracts, affiches, documentaires cinématographiques, enregistrements phoniques, etc. Pour exploiter cette documentation, il faut fixer auparavant les caractéristiques spécifiques du socialisme suisse au XIX ${ }^{\mathrm{e}}$ siècle afin d'identifier dans la documentation le socialisme de qui ne l'est pas. Pour fixer les caractéristiques et les contours de ce phénomène dit socialisme, on combinera une série d'articles de foi, de normes,

98 Sur ces deux auteurs reste fondamental le livre de F. Venturi, Jean Jaurès e altri storici della Rivoluzione francese, Torino, Einaudi, 1948.

99 I. Deutscher, The Prophet Armed. Trotsky: 1879-1921; The Prophet Unarmed. Trotsky: 1921 1929; The Prophet Outcast. Trotsky: 1929-1940, et du même également La révolution inachevée. Cinquante années de révolution en Union Soviétique, 1917-1967, Paris, Laffont, 1967.

100 A. Dansette, Mai 1968, Paris, Plon, 1971.

101 A. Touraine, Le mouvement de mai ou le communisme utopique, Paris, Seuil, 1968. 
de coutumes, de préceptes, de conduites, de notions, de règles, de significations, le parti, l'organisation de classe, et on les constituera en un arrangement idéalisé. Ensuite, on comparera les traces, les indices, la documentation disponible avec le type idéal; l'analyse portera sur la convergence, la divergence ou le parallélisme des matériaux historiques et la construction typologique. On sait que, selon la définition classique de Max Weber: «Un type idéal est formé à partir de l'accentuation d'un ou plusieurs points de vue, et par la synthèse d'un grand nombre de phénomènes individuels concrets, diffus, discrets, plus ou moins présents, qui sont organisés en accord avec ses points de vue mis en relief pour former une construction analytique unifiée. Dans sa pureté conceptuelle, cette construction mentale en effet ne se retrouve nulle part dans la réalité.» C'est pour cela que Karl Marx ne parle pas de capitalisme, mais de capital. A défaut d'une bonne maîtrise théorique des types idéaux, il y a le risque de décrire des réalités qui n'existent pas, d'homogénéiser des situations contrastantes, de parler du socialisme de Thomas Campanella, de capitalisme mérovingien, de précapitalisme, et ainsi de suite.

L'utilisation correcte des instruments théoriques, des typologies, améliore notablement approche du chercheur et lui sert de garde-fou contre les distorsions inhérentes à toute conceptualisation. Savoir manier une théorie, c'est aussi assurer la bonne formulation des hypothèses, d'hypothèses riches, susceptibles d'épouser davantage le réel dans ses contradictions.

L'historien pourrait emprunter au sociologue d'autres classes importantes de théories et parmi celles-ci, notamment, celles de modèle et de structure.

Le modèle est une théorie particulière, hypothético-déductive. En histoire économique, on utilise le mot modèle dans le sens de module ou de schéma. Les exemples les plus courants sont les modèles des cycles économiques, ou les mouvements de longue durée de Kondratieff ${ }^{102}$. Les modèles des cycles servent à expliquer les oscillations entre la phase ascendante du phénomène, qui est beaucoup plus longue, et la phase descendante qui est extrêmement courte. Pour expliquer ce phénomène, l'historien économiste doit recourir à une hypothèse formulée ainsi: les investissements, l'épargne, la consommation, bref les quantités économiques globales sont liées de façon instantanée ou différée. Si les données empiriques vérifient cette hypothèse, le modèle est jugé pertinent pour éclaircir les cycles.

Le modèle par rapport à la théorie a ceci de particulier: tandis que la théorie doit uniquement servir à éclairer la nature d'un phénomène, le modèle doit être en principe toujours vérifiable. L'exemple le plus célèbre est celui de Pareto ${ }^{103}$, dont le modèle a été appliqué à des domaines fort différents, de l'étude de la distribution des mots dans le roman Ulysse de James Joyce, au jeu de billes, à l'inégalité des revenus et à tant d'autres domaines. Son auteur a commencé par

${ }_{102}$ G. Imbert, Des mouvements de longue durée Kondratieff, Aix-en-Provence, La pensée universitaire, 1959, et A. Agnati, Sul ciclo di Kuznets, «Giornale degli Economisti e Annali di Economia », novembre-dicembre 1970, pp. 906-921

103 V. Pareto, Distribution de la richesse d'après le rôle de la taille, à Paris, en 1201, in « Journal de la Société de Statistique de Paris », juillet 1900, pp. 224-225, et du même auteur les Ecrits sur la courbe de la répartition de la richesse, réunis et présentés par G. Busino, Genève, Droz, 1967, ainsi que les Ecrits épars. Textes réunis avec une introduction par G. Busino, Genève, Droz, 1974. 
l'appliquer à la distribution de la richesse dans les villes italiennes du Moyen Age, en Prusse, à Bâle, à Zurich, puis il a voulu le vérifier en l'appliquant à la distribution de la richesse d'après le rôle de la taille, à Paris, en 1292. Dans cette étude Pareto montre que la «distribution de la richesse n'a pas beaucoup changé, à Paris, de 1292 à nos jours. L'exposant $a$, notamment, avait, en 1292, la valeur de 1,37 , il avait, en 1900, la valeur de 1,42.» Des immenses changements sociaux et économiques séparent Paris du début du siècle $X^{\mathrm{e}}$ du Paris de 1292. Ce résultat n'en est plus que «très remarquable». Sans entrer dans trop des détails, on peut dire que le modèle de Pareto met en lumière qu'à toutes les époques, la répartition du revenu affecte la forme d'une toupie dont la pointe est tournée vers le haut, et cela dans des pays de conditions économiques et sociales fort différentes. Pareto dit que cette forme ne dépend pas du hasard car si tel était le cas, elle serait semblable à la courbe des probabilités. Or, la courbe de la répartition diffère totalement de celle des probabilités, bien connue des statisticiens, sous le nom de «courbe des erreurs ». Pareto conclut que la forme, une sorte de toupie renversée, est engendrée par des forces fondamentales indépendantes, gouvernées par une loi universelle. Les pauvres constituent la partie inférieure, arrondie, de la toupie renversée, et les riches le sommet, en pointe. Le modèle permet de constater, en outre, qu'une augmentation des revenus minima et une diminution de l'inégalité ne peuvent se produire, soit isolément soit d'une manière cumulative, que si le total des revenus croît plus vite que la population; l'accroissement du nombre des grandes fortunes n'implique pas un accroissement général de la richesse, pas plus que l'accroissement du nombre des pauvres n'implique un appauvrissement général du pays. En d'autres termes, l'inégalité des fortunes et la diminution du paupérisme sont deux phénomènes sociaux différentes. Par ce modèle, on explique aussi le fait qu'une distribution différente de la richesse n'arrive qu'à élargir la base de la toupie mais pas à amenuiser sensiblement le sommet. En termes plus abstraits, le modèle postule que la courbe de la distribution du revenu est représentée par une droite dont l'équation est $\log N=\log A-a \log x$, où $x$ est la dimension du revenu égal et supérieur à $x, A$ et $a$ étant des constantes à déterminer d'après les données empiriques. L'inclinaison sur l'axe des $x$ de la ligne des logarithmes est donnée évidemment par $a$. Tout cela peut se «voir» grâce à un graphique à double échelle logarithmique où sont représentés les revenus supérieurs à la moyenne; la diminution de l'inclinaison indique une moindre inégalité des revenus.

Le modèle explique ainsi la forme de la distribution de la richesse et des revenus dans toutes les sociétés, à toutes les époques. Dans le Bas-Empire, par exemple, on a pris aux riches des terres et on les a données aux soldats; ailleurs on a établi l'impôt progressif ou généralisé la sécurité sociale. Ces différentes manières de procéder à des redistributions, ont agrandi la base de la pyramide sociale, rendu plus mince le sommet, et pourtant les proportions entre les différentes couches de la société n'ont subi aucune mutation fondamentale. Selon Pareto il y a un équilibre à l'intérieur de la société qui reste toujours le même, d'une époque à l'autre. Dans le Cours d'économie politique, \$1012, il a écrit: «L'inégalité de la répartition paraît donc dépendre beaucoup plus de la nature même des hommes que de l'organisation économique de la société. De profondes modifications de cette organisation pourraient n'avoir que peu d'influence pour modifier la loi de la répartition du revenu.» 
Des inférences putatives font dire à Pareto qu'il y une stabilité de l'ordre social, invariable malgré des répartitions radicales des capitaux et des revenus. Dans ce cas on est allé plus loin de ce que le modèle conseille d'induire. Toutefois, le modèle reste un outil de recherche puissant. Il aide à poser des bonnes hypothèses, à spécifier ensuite les fonctions analytiques, à établir des règles de définition qui sont aussi des règles de transformation. Si le modèle colle avec la réalité, avec les données empiriques, le modèle est validé, il a une certaine utilité cognitive.

Inutile de rappeler que les théories n'existent nulle part dans la réalité. Ce sont des «filets» utilisés pour saisir la complexité du réel. Sans théorie et au vu de la quantité de données à la disposition du chercheur, il serait difficile de commencer une enquête, de classer les matériaux, de les interpréter.

A une autre classe de théorie appartient la structure, avec son cortège des notions de fonction et de signification. La structure est d'une très grande puissance heuristique. En effet, elle aide à expliquer les dynamismes sous-jacents des phénomènes, à élucider les processus d'élaboration et de développement des événements, en ce qu'ils ont d'invariant et d'intemporel. Il suffit de penser au problème de la langue, celle-ci change si lentement que les changements ne sont perceptibles qu'au cours de quelques générations. Un autre exemple nous est donné par la culture, c'est-à-dire par des comportements, des attitudes, par la capacité de profiter d'expériences sociales sans avoir à chaque fois à payer le prix pour l'élaboration de cette expérience. Grâce à la culture l'homme peut résoudre des problèmes qu'il n'avait jusqu'alors jamais affrontés et il peut les résoudre avec une relative aisance et une certaine économie de moyens. Puisqu'il n'y a pratiquement pas d'instincts chez l'homme, la «culture» et l'expérience sociale lui fournissent les moyens de faire face à tous ces problèmes. L'ensemble des comportements hérités, reçus, transmis, c'est la culture, un ensemble d'éléments qui permet de résoudre les problèmes de l'existence. Ces éléments, organiquement liés entre eux, forment une composition, une structure.

Ce concept de structure permet d'aller au-delà de la partie contingente des comportements (de cette partie qui change, qui peut muter) d'aller au tréfonds des événements; il aide à saisir le noyau fondamental d'un phénomène, à aller pardelà les apparences, par-delà le manifeste; il donne au chercheur l'élément pour ainsi dire permanent d'un système, le système d'un objet construit. La structure n'est pas immédiatement observable. Grâce à elle, selon Wittgenstein, «dans l'état des choses, les objets se comportent les uns par rapport aux autres d'une manière déterminée. La manière dont les objets s'enchaînent dans l'état des choses constitue la structure de l'état des choses. La forme est la possibilité de la structure. La structure du fait consiste dans la structure des états de choses » ${ }^{104}$.

On oppose à la notion de structure celle de conjoncture. Si la structure est l'élément permanent, la conjoncture est par contre l'ensemble des variations à court terme d'un certain nombre de variables caractéristiques. En d'autres termes, les structures sont des constantes, et des éléments d'évolution, mais d'une évolution lente. Grâce à la notion de structure, il est possible de repérer les interdépen-

104 L. Wittgenstein, Tractatus logico-philosophicus e Quaderni 1914-1915, Torino, Einaudi, 1968, et surtout les Osservazioni sopra i fondamenti della matematica, Torino, Einaudi, 1971 
dances des modes de production, des hiérarchies sociales, des institutions et des croyances. Ce concept, bien manié, arrive à rendre compte des décalages, des interdépendances qui n'apparaissent pas, de la production de structures nouvelles, des inadaptations de niveaux et des équilibres dans le sens des régulations et des autorégulations ${ }^{105}$. Mais pour y parvenir, il faut savoir comment construire cette notion abstraite, cette théorie, autrement dit, la structure, dont Claude LéviStrauss a dit: «Les structures sociales sont des objets indépendants de la conscience qu'en prennent les hommes (dont elles règlent pourtant l'existence) et sont aussi différentes de l'image qu'ils s'en forment que la réalité physique diffère de la représentation sensible que nous en avons ou des hypothèses que nous formulons à ce sujet ${ }^{106}$. De ce fait, il faut aller au-delà de la pure observation descriptive - et c'est bien grâce à cette rupture épistémologique que Les structures élémentaires de la parenté, par-delà la compréhension des formes du lien familial, et des échanges matrimoniaux, aident à comprendre les échanges sociaux, les fonctions sociales et les institutions, et en somme tous les rapports et les liens d'association et de subordination entre les hommes. Par-delà les structures matrimoniales, ce livre a révélé que là où il y a un système de parenté ${ }^{107}$, il s'établit inévitablement une structure hiérarchique, et là où il $\mathrm{y}$ a une structure hiérarchique, il y a toujours un rapport de subordination et de domination. Il faut ajouter qu'assez souvent les structures se présentent à nous sous une forme symbolique. En général, c'est le symbole qui médiatise les rapports de domination et de subordination. Par exemple, les messages, les signaux, la manière de parler permettent d'assurer pour ainsi dire l'hégémonie, les rapports de subordination-domination qui sont inhérents à la nature humaine, telle tout au moins que nous l'avons connue jusqu'ici.

En plus, donc, des apports théoriques, la sociologie peut fournir à l'histoire une panoplie de techniques et de méthodes. Elles sont fort nombreuses, je ne saurais les énumérer toutes. Il va de soi que les techniques sociologiques ne fournissent jamais à l'histoire des solutions toutes faites. Toutefois, elles peuvent aider à poser de manière nouvelle des problèmes anciens ou plus simplement à indiquer de nouvelles voies d'approche.

Prenons deux exemples: l'analyse des contingences, et les sondages par panels. L'analyse des contingences est une technique permettant d'établir des structures de relations entre les éléments d'un message. Grâce à cette technique, nous arrivons à préciser les symboles-clés d'une campagne électorale et ensuite à calculer la fréquence à laquelle chaque symbole-clé apparaît avec d'autres symboles-clés. Il faut tout d'abord regrouper les symboles réputés importants en catégorie et ensuite aller de l'avant grâce au calcul matriciel. Je n'entre pas dans les détails techniques - que d'ailleurs nous pouvons même ignorer ici. C'est bien cette analyse des contingences qui a permis de disséquer le journal de Goebbels, en utilisant 21 catégories. On est arrivé à construire une matrice pouvant rendre

\footnotetext{
105 J. Piaget, L. Apostel et B. Mandelbrot, Logique et équilibre, Paris, PUF, 1957, p. 46. Les régulations sont des compensations partielles qui ont pour effet de modérer les déformations (= transformations non compensées), par rétroaction ou par anticipation.

106 Cl. Lévi-Strauss, Anthropologie structurale, Paris, Plon, 1958, chap. XV.

${ }_{107}$ Cl. Lévi-Strauss, Les structures élémentaires de la parenté, Paris, Mouton, 1968.
} 
compte de la présence / absence des catégories dans tous les passages du journal. On s'est aperçu alors qu'il y a des catégories corrélées très strictement du point de vue statistique - et dans ce cas, chose un peu étrange qu'on a eu de la peine par la suite à expliquer, elles étaient toujours associées psychologiquement dans la pensée de Goebbels. Par exemple, l'analyse montra que les Juifs, les Italiens et les Français étaient pour Goebbels des peuples d'esclaves. Pourtant, cette idée s'associe à celle d'assurer des réserves alimentaires à un seul de ces pays, la France. Et cette idée à son tour s'accompagne toujours de celle de la supériorité allemande en toutes circonstances et en particulier vis-à-vis des Français. Il a été aisé de présenter alors la structure du journal graphiquement et ainsi en avoir une synthèse visuelle ${ }^{108}$. Une telle technique est très utile lorsqu'on a affaire à une masse considérable de documents, ou lorsqu'il est impossible à un seul chercheur de maîtriser des milliers et des milliers de pages de mémoires ou de correspondance.

L'autre exemple concerne les enquêtes par sondage. L'enquête est menée sur un échantillon construit selon les méthodes scientifiques de la représentativité et du plan d'échantillonnage. En sociologie, le sondage est un moyen très courant pour recueillir les opinions exprimées par les personnes appartenant à une population finie ou infinie. Il est utilisé pour connaître l'opinion des Suisses sur les Hautes Ecoles spécialisées, ou celle des Italiens à propos de la mainmise de la mafia sur les travaux publics, ou encore des Anglais au sujet des prestations du service national de santé. Il va de soi que le sondage donne aussi des résultats pour l'analyse de phénomènes plus complexes, par exemple les phénomènes électoraux. Ceux qui étudient les élections savent l'importance des documents administratifs pour ce genre de recherches. Grâce à ces documentations, on arrive à connaître la composition socioprofessionnelle des villes, et grâce au dépouillement des votes, on peut connaître la répartition des votes par quartier et par commune. Ces deux types de renseignements restent, il est vrai, toujours juxtaposés car ces deux sources différentes ne donnent aucune indication sur la relation entre le vote et la catégorie socioprofessionnelle. Il est difficile de dire si les suffrages du parti socialiste proviennent des milieux ouvriers et ceux du parti radical de la petite bourgeoisie, par exemple. Cette difficulté est contournée à l'époque contemporaine par les sondages. Les sondages font en effet connaître les votes passés, évidemment à condition que les déclarations fournies à l'enquêteur soient vraies. Les renseignements fournis par les sondages sociologiques ne sont pas du tout juxtaposés, mais corrélés entre eux. Ils font connaître l'opinion publique, sa constitution, ses tergiversations, ses atermoiements ainsi que les processus de formation des décisions électorales ${ }^{109}$. En général, on connaît le

108 Tiré de I. De Sola Pool, Content Analysis Today: A Summary, in Trends in Content Analysis, Urbana, University of Illinois Press, 1959, p. 37. Pour des observations critiques très importantes, cf P. Henry \&. S. Moscovici, Problèmes de l'analyse de contenu, «Langages », septembre 1968, n. 11, pp. 36-60, ainsi que Influencia manifesta e influencia oculta en la comunicacion, « Revista mexicana de Sociologia», 45, 1983, n. 2, pp. 687-701.

109 Cf. F. Carpentier, Faut-il publier les sondages d'opinion?, «Economies et sociétés», décembre 1970, pp. 2353-2364; P. Bourdieu, Les doxosophes. A propos des sondages d'opinion en matière politique, «Minuit», novembre 1972, 26-45; J. Stoetzel, Faut-il brûler les sondages?, «Preuves», n. 13, pp. 7-14; P. Champagne, Faire l’opinion. Le nouveau jeu politique, Paris, Les 
résultat du vote, mais on ne peut rien dire de la façon dont on est arrivé à ce résultat, sinon de manière plus ou moins impressionniste. Dans ce domaine, en plus, les sociologues ont mis au point des techniques extrêmement perfectionnées de sondage, qui peuvent être d'une très grande utilité pour les historiens, telle cette forme particulière de sondage qu'est l'enquête par panel ${ }^{110}$, consistant à interroger à plusieurs reprises un échantillon de sujets en éliminant avec des procédés mathématiques les distorsions naissant de la répétition de la même expérience. Supposons d'avoir 4 vagues d'interviews et que toutes les questions posées visent à mettre en évidence 3 caractéristiques : avoir des informations sur le degré d'intérêt pour la consultation électorale (fort ou faible), sur le degré d'exposition volontaire à la campagne électorale (fort ou faible selon qu'on fréquente des meetings ou qu'on allume la télévision pour entendre les discours de M. Vincent ou de M. Reverdin, selon qu'on lit les journaux lorsqu'ils relatent le débat au sujet d'une votation); enfin, sur les caractéristiques du vote, c'est-à-dire voter ou ne pas voter. J'ai simplifié au maximum en choisissant uniquement 3 caractéristiques.

Ces caractéristiques se présentent sous la forme de variables à deux états : fort ou faible, voter ou s'abstenir. Nous avons des facteurs constitutifs dont la composition et les rapports déterminent la situation considérée et qui peuvent prendre diverses valeurs, dites valeurs ordinales (plus grand que / égal / plus petit que). On peut introduire des variables plus complexes, mais alors dans ce cas-là, il faut avoir un ordinateur, étant donné que les calculs deviennent extrêmement compliqués. Au moment de la première entrevue-observation, les réponses des interviewés peuvent être classées $2 \times 2 \times 2=8$, c'est-à-dire qu'elles peuvent nous donner 8 caractéristiques différentes, c'est-à-dire 8 réponses différentes à notre question. De même à la seconde, à la troisième et à la quatrième interview. Nous disposons à la fin de 4096 informations numériques, c'est-à-dire 4096 informations au sujet de cet échantillon que l'on a interviewé 4 fois. Nous savons que certains sujets peuvent être classés +++ , intérêt fort, exposition forte, vote positif à la première observation, +-+ à la deuxième, et ainsi de suite pour toutes les combinaisons possibles et imaginables. Or, une fois que nous avons ces observations et les exploitons pour construire un tableau, nous allons d'un coup découvrir un processus que nous n'aurions jamais pu saisir autrement. Nous décelons un processus gouverné par un réseau causal complexe, qu'il était difficile d'entrevoir avant, c'est-àdire que l'intérêt politique provoque la recherche de l'information et que ces deux facteurs solidairement tendent à éliminer l'attitude d'abstentionnisme. Il est donc prouvé qu'une propagande extrêmement poussée n'arrivera pas à éliminer l'abstentionnisme qui, dans ce pays, est particulièrement important. Ceux qui pensent qu'avec un peu de propagande, on éliminera l'abstention-

Editions de Minuit, 1990. Voir aussi J. Padioleau, L'opinion publique. Examen critique, nouvelles directions. Recueil de textes, Paris, Mouton, 1981; E. Noelle-Neumann, The spiral of Silence. Public Opinion-Our Social Skin, Chicago, University Press, 1993 et Natur des Meschen. Beiträge zur empirischen Kommunikationsforschung, Allensbach a. B., Aber, 2002.

110 F. Chazel, R. Boudon et P. Lazarsfeld, L'analyse des processus sociaux, Paris-La Haye, Mouton, 1970, pp. 177-360. 
nisme, risquent de s'attaquer à un phénomène superficiel; la solution de ce problème superficiel ne changera strictement rien à cette réalité-là ... à une réalité extrêmement profonde si bien décrite, il y a plus d'un siècle, par Tocqueville. On pourrait ajouter un autre exemple un peu plus complexe, un peu plus classique aussi, élaboré par James Coleman, reconstruit par Paul-F. Lazarsfeld et repris récemment par Raymond Boudon ${ }^{111}$. On peut le résumer en l'adaptant pour vite voir comment certaines techniques sociologiques peuvent donner aux historiens une aide précieuse.

Dans une élection, on a observé les résultats suivants:

$\begin{array}{lrrrrrrrrrr}N & & & & & & & & \\ 2 & 30 & 20 & 110 & & & & & \\ 3 & 320 & 150 & 300 & 520 & & & & \\ 4 & 220 & 130 & 200 & 260 & 620 & & & \\ 5 & 160 & 70 & 150 & 130 & 200 & 240 & & \\ 6 & 80 & 80 & 190 & 140 & 170 & 180 & 210 & \\ 7 & 40 & 70 & 130 & 140 & 120 & 120 & 130 & 200 \\ \ldots & \ldots & \ldots & \ldots & \ldots & \ldots & \ldots & \ldots & \ldots & \\ & 1 & 2 & 3 & 4 & 5 & 6 & 7 & \ldots & j\end{array}$

Le cas est fictif; en général, on a des tableaux plus longs et complexes.

$N$ est l'effectif des bureaux de vote dans le canton de Vaud; $j$ représente le nombre de personnes ayant voté pour le candidat Bolomey - comment devonsnous lire ce tableau?

Il se lit de la manière suivante: parmi les $30+20+110=160$ sections de deux membres, 30 ont voté à l'unanimité pour le candidat Chapuy opposé à Bolomey, 110 ont voté à l'unanimité pour le candidat Bolomey, tandis que dans vingt sections les deux voix se partagent sur chacun des candidats. Dans les sections de trois membres, dans 320 cas, les trois membres votent pour Chapuy, dans 520 cas les trois membres votent pour Bolomey, dans 150 cas, un membre vote pour Bolomey et deux pour Chapuy. Dans 300 cas, un membre vote pour Chapuy et deux pour Bolomey.

Continuer cette lecture n'est pas nécessaire. Ce qui est important, dans ce tableau, saute vite aux yeux: il y a unanimité ou quasi-unanimité dans le comportement des bureaux électoraux. Le tableau fait apparaître, en effet, l'existence d'un processus souterrain, que Coleman et Lazarsfeld ont bien élucidé, c'est-àdire que le vote est influencé par la répartition des voix à l'intérieur du bureau de vote. C'est une banalité désormais, depuis qu'on s'occupe de comportement électoral, que d'affirmer cela; et pourtant, il y a 50 ans, personne n'y songeait encore.

${ }^{111}$ Op. cit. On aurait pu citer également l'étude de M. Gary et J.W. Hilty Fink, Metodi statistici e storia : il «New Deal» di Truman attraverso le sue dichiarazioni di voto, « Rassegna economica», 1972, fasc. III, pp. 513-612. 
Prenons maintenant la technique du panel. Elle est appliquée couramment à l'histoire, notamment en Amérique. Parmi les tout derniers livres qui ont utilisé la technique du panel, il faut citer celui que Martin Seymour Lipset a consacré à l'élection de 1860 aux U.S.A., au référendum sur la sécession des Etats du Sud des Etats-Unis. Les résultats ont littéralement renouvelé cette page extrêmement importante de la vie politique américaine, et même socio-économique, étant donné qu'au cours de cette élection de 1860, il y a eu un brassage entre le parti whig et le parti tory, brassage qui a pratiquement chambardé toute la carte politique des Etats-Unis, en lui donnant l'empreinte que nous lui voyons aujourd'hui encore $^{112}$. Cette technique pourra renouveler l'histoire politique, pourra donner une dimension totalement nouvelle aux études d'histoire religieuse, ou d'histoire intellectuelle.

Il ne reste qu'à dire quelques mots sur l'importance des recherches sociologiques pour l'historien de demain. Chaque année, la corporation des sociologues publie environ 10.000 études, sans compter les travaux destinés à une circulation limitée, sous forme de documents ronéotypés et qui, dans nos bibliothèques, occupent davantage de place que les livres. Le moment est venu pour les bibliothèques ou tout au moins pour les instituts d'histoire de commencer à s'intéresser à cette littérature grise, à la classer et à la mettre à la disposition des chercheurs. Car dans ces rapports de recherches, l'historien de demain trouvera, pour autant qu'il saura les lire, tous les éléments pour comprendre notre société complexe et compliquée; il y trouvera déjà faite, au moins en partie, l'histoire de ce présent si fascinant. L'histoire de demain trouvera dans les recherches des sociologues d'aujourd'hui des données importantes surtout dans les domaines de la stratification sociale, de la mobilité, des comportements collectifs, de la socialisation et de la déviance, de l'opinion publique. La célèbre recherche sur l'invasion des Martiens est un document désormais classique sur les croyances collectives générées par de fausses nouvelles médiatiques.

La belle émission d'Orson Wells sur l'invasion de l'Amérique par les Martiens avait pratiquement paralysé la vie quotidienne. Les Américains imaginaient que les Martiens étaient arrivés sur la Planète bleue. Le phénomène avait eu une telle ampleur que des dizaines de chercheurs ont voulu connaître les raisons de ce cas de panique collective. Comment se faisait-il que, dans un pays qui avait le meilleur niveau culturel du monde, qui était à l'avant-garde de la science et de la technologie, où la laïcisation de la vie était extrêmement poussée, où il y avait un degré de connaissances astronomiques parmi les plus approfondies, on ait pu assister à une forme d'hystérie collective, de panique généralisée. Des historiens se sont occupés de ce problème à partir des chroniques et des commentaires des journalistes, de mémoires d'acteurs, de récits d'écrivains. Ils ont établi la chronologie de l'événement et en ont tracé l'histoire telle qu'elle a été rationalisée a posteriori. Les historiens, en relatant les opinions exprimées, se fondaient sur des types de rationalisation construite a posteriori; ils reflétaient bien évidemment les idées d'une couche particulière de la population, celle qu'on appelle «l'opinion

112 S.M. Lipset, Political Man, The Social Bases of Politics, New York, Doubleday, 1960, et du même Les préjugés dans l'Amérique d'hier et d'aujourd'hui, «Sociologie et Sociétés», mai 1969, pp. 105-134. 
publique américaine». Les sociologues et psychologues sociaux, par contre, ont étudié l'invasion des Martiens avec d'autres techniques. Ces travaux expliquent l'impact que l'annonce d'une invasion de la terre par les Martiens a eu aux EtatsUnis et aident également à comprendre les comportements réels de diverses strates de la population américaine tout de suite après la Deuxième Guerre mondiale. A l'aide de ces enquêtes psychosociologiques, les historiens disposent d'une compréhension rapprochée de la panique telle qu'elle s'était diffusée dans les divers groupes sociaux ${ }^{113}$.

J'ai souligné plus haut l'importance pour les historiens des travaux sociologiques sur la stratification sociale. Un seul exemple ici suffira. On a dit et répété que la Suisse est une société sans classes. Cette affirmation, on la retrouve dans tous les livres d'historiens suisses. Cette thèse se fonde sur des indicateurs assez importants tels que les salaires, le statut socioprofessionnel, le degré d'intégration (mais un récent sondage, fait à l'occasion du 1er mai, a montré que les Suisses ne sont pas ce peuple si attaché à la participation tel qu'on l'imaginait). Bref, l'affirmation repose sur un certain nombre d'indicateurs apparemment très sûrs. Ces indicateurs démontreraient, d'après les historiens, que le pays n'a pas de classes sociales. Or, si, au lieu de s'en tenir aux apparences exprimées par ces indicateurs sociaux, on examinait les enquêtes sociologiques en la matière, on trouverait de bien curieuses choses. Lorsqu'on a demandé aux Suisses la classe à laquelle ils pensent appartenir, les ouvriers sont deux fois moins nombreux $(36 \%)$ à se ranger parmi les travailleurs. Les petits ou les ouvriers, selon les termes utilisés, se déclarent dans la proportion de $61 \%$ membres des classes moyennes ou de la petite bourgeoisie. Par ailleurs, nous savons que parmi les cadres supérieurs de ce pays et les membres des professions libérales, la proportion de ceux qui se disent des classes moyennes et qui refusent de se considérer des bourgeois est à peu près aussi forte $(82 \%)$ que parmi les employés $(85 \%)$.

N'est-il pas évident que la proclamation de l'appartenance aux classes moyennes n'a pas et ne peut avoir la même signification pour les membres des différentes catégories sociales? Luc Boltanski, dans son livre reçu comme un pamphlet déplacé, Le Bonheur suisse, a très pertinemment observé: «Ce qui est parade chez les ouvriers ne peut être que feinte dans les classes supérieures, sacrifice intéressé à la passion égalitaire par laquelle, tout à la fois, on peut garder ses distances et décourager la revendication en déniant la réalité du privilège ou de l'existence des privilèges $»^{114}$.

$\mathrm{Si}$ nous prenons maintenant en considération les travaux de sociologie de l'éducation et les lisons en fonction d'une étude de mobilité sociale, c'est-à-dire si nous faisons une analyse secondaire de ces travaux, nous découvrons qu'au-delà d'un même idéal, celui d'une société méritocratique, basée sur l'idéologie de l'effort commun, de l'attachement aux valeurs démocratiques et aux vertus d'égalité, par-delà tout ce qui fait de ce pays « un des meilleurs pays qui soit » (la phrase est de Vilfredo Pareto), nous constatons qu'en Suisse il y a des classes, et que ces classes ont des possibilités différentielles d'accès à la richesse, au savoir et aux

${ }^{113}$ H. Cantril, The Psychology of Social Movements, New York, Wiles, 1941, et P. F. Lazarsfeld, On Social Research and Its Language, Chicago, University of Chicago Press, 1993.

114 L. Boltanski, Le bonheur suisse, Paris, Minuit, 1966. 
loisirs. Dès lors, le sociologue constate un décalage entre les normes de vie que propose la culture nationale et les comportements imposés aux individus par les conditions objectives dans lesquelles ils se meuvent. Est-ce que ce décalage entre le réel et l'idéal engendre ce que l'on a pris l'habitude d'appeler le «mal suisse» ou «l'histoire d'un peuple heureux », pour le dire d'une autre manière? Voici une question à laquelle les historiens pourraient très facilement répondre pour autant qu'ils s'intéressent davantage aux travaux sociologiques qu'aux actes parlementaires et aux registres des décisions du Conseil fédéral.

\section{EN GUISE DE CONCLUSION INTERLOCUTOIRE}

La conclusion, je veux l'emprunter aux Problèmes généraux de la recherche interdisciplinaire de Jean Piaget: «Inutile de multiplier les exemples pour montrer qu'il y a là un domaine de recherche interdisciplinaire d'importance assez générale. Le problème revient en définitive à se demander jusqu'à quel point l'homme contemporain dépend de son histoire. Une réponse superficielle qui pourrait être tirée de ce que l'on vient de voir consisterait à soutenir que les facteurs historiques ont d'autant plus d'importance qu'ils sont intemporels et relèvent, comme les normes rationnelles, d'invariants que l'histoire retrouve mais ne crée ou n'explique pas, tandis que les grands changements historiques qui introduisent des continuités entre certains systèmes de normes et les précédents souligneraient davantage l'importance des rééquilibrations synchroniques que des processus constructeurs continus. En réalité, il y a l'histoire des événements, ou des manifestations visibles et en partie contingentes, et il y a l'histoire du dynamisme sousjacent ou des processus d'élaboration et de développement. Or, on sait de plus en plus qu'un développement organique est bien davantage qu'une histoire d'événements ou qu'une succession de phénomènes: il est structuration ou organisation progressive, dont les étapes qualitatives sont subordonnées à une intégration croissante. C'est pourquoi l'histoire de la civilisation est de plus en plus une œuvre interdisciplinaire où l'histoire des sciences et des techniques, l'histoire économique, la sociologie diachronique, etc., doivent analyser concurremment les innombrables faces des mêmes transformations, mais aussi pourquoi l'histoire est explicative même en ce qui apparaît comme des invariants intemporels, car ils ne sont devenus tels qu'en fonction de processus constructifs et d'équilibration qu'il s'agit de reconstituer et qui, en opérant d'un domaine à l'autre, s'éclairent les uns les autres en leurs oppositions autant qu'en leurs mécanismes communs.» ${ }^{115}$

Genève

115 J. Piaget, Problèmes généraux de la recherche interdisciplinaire et mécanismes communs, in Tendances principales de la recherche dans les sciences sociales et humaines. Première partie: Sciences sociales. Préface de R. Maheu, Paris, Unesco, 1970, pp. 588-589. 